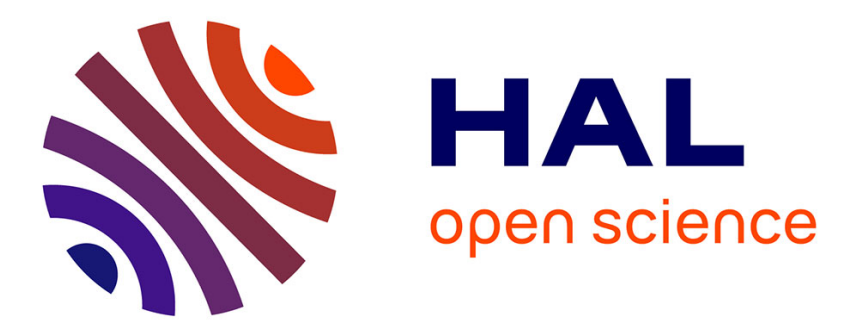

\title{
Detection of Jupiter decametric emissions controlled by Europa and Ganymede with Voyager/PRA and Cassini/RPWS
}

\author{
C. Louis, L. Lamy, P. Zarka, B. Cecconi, S. L. G. L G Hess
}

\section{> To cite this version:}

C. Louis, L. Lamy, P. Zarka, B. Cecconi, S. L. G. L G Hess. Detection of Jupiter decametric emissions controlled by Europa and Ganymede with Voyager/PRA and Cassini/RPWS. Journal of Geophysical Research Space Physics, 2017, 10.1002/2016JA023779 . hal-01599667

\section{HAL Id: hal-01599667 \\ https: / hal.sorbonne-universite.fr/hal-01599667}

Submitted on 3 Oct 2017

HAL is a multi-disciplinary open access archive for the deposit and dissemination of scientific research documents, whether they are published or not. The documents may come from teaching and research institutions in France or abroad, or from public or private research centers.
L'archive ouverte pluridisciplinaire HAL, est destinée au dépôt et à la diffusion de documents scientifiques de niveau recherche, publiés ou non, émanant des établissements d'enseignement et de recherche français ou étrangers, des laboratoires publics ou privés. 


\section{Journal of Geophysical Research: Space Physics}

\section{RESEARCH ARTICLE}

10.1002/2016JA023779

\section{Key Points:}

- Detection of Jupiter decametric emissions controlled by Europa and Ganymede

- Average properties of the Europa-DAM emissions (spectral, temporal variability, maximal occurrences, and active longitudes)

- Average properties of the Ganymede-DAM emissions (spectral, temporal variability, maximal occurrences, and active longitudes)

Correspondence to:

C. K. Louis,

corentin.louis@obspm.fr

Citation:

Louis, C. K., L. Lamy, P. Zarka, B. Cecconi, and S. L. Hess (2017), Detection of Jupiter decametric emissions controlled by Europa and Ganymede with Voyager/PRA and Cassini/RPWS, J. Geophys. Res. Space Physics, 122 , doi:10.1002/2016JA023779.

Received 20 DEC 2016 Accepted 18 JUL 2017

Accepted article online 15 AUG 2017

O2017. American Geophysical Union. All Rights Reserved.

\section{Detection of Jupiter decametric emissions controlled by Europa and Ganymede with Voyager/PRA and Cassini/RPWS}

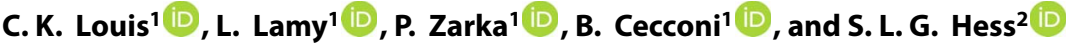 \\ ${ }^{1}$ LESIA, Observatoire de Paris, PSL Research University, CNRS, Sorbonne Universités, UPMC Université Paris 06, Université \\ Paris Diderot, Sorbonne Paris Cité, Meudon, France, ${ }^{2}$ Department of Space Environment, ONERA-The French Aerospace \\ Lab, Toulouse, France
}

Abstract The Jovian high-latitude radio emissions produced by Jupiter's magnetosphere extend from a few kilohertz to $40 \mathrm{MHz}$. Part of the decametric (DAM) emissions are driven by the Galilean moon lo (Io-DAM). As UV aurorae have been detected at the footprint of Europa and Ganymede, we expect that these moons drive Jovian radio emissions as well. To check this assumption, we used the ExPRES simulation code (Exoplanetary and Planetary Radio Emissions Simulator) to predict dynamic spectrum (time-frequency spectograms) of the radio emissions controlled by the four Galilean moons. Then we compared the simulations to the Voyager/PRA and Cassini/RPWS radio observations of Jupiter (1979, and between 2000 and 2003, respectively). We present the first clear evidence for the existence of decametric emissions controlled by Europa and Ganymede. Their statistical analysis allows us to describe the average properties of the Europa-DAM and Ganymede-DAM emissions such as their spectrum, temporal variability, and occurrence as a function of moon phase and subobserver's longitude.

\section{Introduction}

Radio emissions produced by Jupiter's magnetosphere between a few kilohertz and $40 \mathrm{MHz}$ have been studied for half a century. Jovian auroral radio emissions are thought to be produced through the Cyclotron Maser Instability (CMI), at a frequency close to the electron cyclotron frequency, from non-Maxwellian weakly relativistic electrons spiraling along high-latitude magnetic fields lines [see Zarka, 1998, Treumann, 2006, and references therein]. These emissions are produced in the auroral regions above the atmosphere by non-Maxwellian electron distribution function, along a thin hollow conical sheet at large angle from the local magnetic field. They are elliptically polarized, the ones originating from the northern hemisphere are mainly right handed $(\mathrm{RH})$, whereas the southern ones are mainly left handed $(\mathrm{LH})$. These emissions consist of several spectral components: the kilometric (KOM), hectometric (HOM), and decametric controlled by lo (lo-DAM) or not (non-lo-DAM).

The lo-DAM was first identified by Bigg [1964]. lo emissions appear as four main types of arcs lasting for a few hours in time-frequency spectrograms. They have been historically labeled as lo- $A, B, C$, and D depending on the position of the source, each with different characteristics: this is due to radio beaming anisotropy [Hess et al., 2014]. A and B are northern emissions (RH polarization), while C and D are southern emissions (LH polarization). A and $C$ are emitted eastward of Jupiter and generally observed as closing parentheses (so-called Vertex Late (VL) arcs). B and D are emitted westward and generally observed as opening parentheses (so-called Vertex Early (VE) arcs) [see Carr et al., 1983; Marques et al., 2017 (Figure 2)]. An auroral spot at the footprint of lo was later discovered in the infrared (IR) by Connerney et al. [1993] and in the ultraviolet (UV) by Prangé et al. [1996] and Clarke et al. [1998].

The UV footprints of Europa and Ganymede were more recently detected by Clarke et al. [2002]. The interaction between Jupiter and these moons was therefore expected to produce radio emission similar to lo-DAM.

Signatures of radio emissions induced by Ganymede, Europa, and Callisto have been tentatively identified in Galileo/PWS and Voyager/PRA radio observations between $\simeq 2.0$ and $\simeq 5.8 \mathrm{MHz}$, over 2 years for Galileo/PWS and 4 months for Voyager/PRA 1 and 2, as briefly reminded below. 
Menietti et al. [1998a] analyzed direction-finding results of Galileo/PWS and identified two arcs which could be consistent with emission driven by Ganymede. Menietti et al. [1998b], alternately analyzed Galileo/PWS data between subobserver's longitudes (Central Meridian Longitude, CML) $100^{\circ}$ and $160^{\circ}$ as a function of Ganymede phase and observed a modest enhancement of emission occurrence around $80^{\circ}$ and $245^{\circ}$ in terms of the phase of Ganymede. The phase of a satellite increases with the orbit of the moon, with $0^{\circ}$ when Jupiter lies directly between it and the observer (superior conjunction) [see Marques et al., 2017, Figure 1]. Hospodarsky et al. [2001] reanalyzed the same Galileo data with a different method and observed a similar weak enhancement of the emission occurrence over the same range of Ganymede's phase than Menietti et al. [1998b].

Higgins et al. [2006] organized the Galileo/PWS data as a function of Europa phase and CML. They identified an enhancement of the emission occurrence in two different regions. On the northeast side of Jupiter $\left(\left[290^{\circ}-320^{\circ}\right] \mathrm{CML} ;\left[260^{\circ}-290^{\circ}\right]\right.$ Europa phase) and on the northwest side $\left(\left[90^{\circ}-180^{\circ}\right] \mathrm{CML} ;\left[90^{\circ}-110^{\circ}\right]\right.$ Europa phase).

Menietti et al. [2001] tentatively observed Callisto-induced emissions using the Galileo/PWS data. They found an enhancement in the emission occurrence near phases of $80^{\circ}$ and $260^{\circ}$.

Using Voyager/PRA data, Higgins [2007] reported a minor excess of emission occurrence at 95\% confidence level in the $C M L$ range $\left[290^{\circ}-350^{\circ}\right]$, at orbital phase $\left[85^{\circ}-115^{\circ}\right]$ and $\left[250^{\circ}-280^{\circ}\right]$ for Europa, $\left[55^{\circ}-85^{\circ}\right]$ and $\left[305^{\circ}-335^{\circ}\right]$ for Ganymede, and $\left[105^{\circ}-135^{\circ}\right]$ and $\left[300^{\circ}-330^{\circ}\right]$ for Callisto.

To check and confirm the existence of DAM emissions induced by Ganymede, Europa, and Callisto, we used a different approach. Our study uses the radio emission simulator ExPRES [Hess et al., 2008, section 2] which allows us to search for Jupiter decametric emissions controlled by lo, Europa, Ganymede, and Callisto in the Cassini/RPWS and Voyager/PRA radio observations of Jupiter. In section 2, we present the ExPRES simulations, the set of observations to which they are compared, and the detection criteria chosen to identify the Jupiter satellite emissions. In section 3, we present the results, and in section 4 we analyze statistically the detections. In section 5 we discuss these results.

\section{Methodology}

\subsection{EXPRES}

The Exoplanetary and Planetary Radio Emissions Simulator (ExPRES) computes the geometrical visibility of radio sources around a magnetized planet and tests at each time/frequency step whether the radiated waves are visible or not for a given observer. These results are then used to produce time-frequency spectrograms (or dynamic spectrum) of visible radio sources which can be directly compared to observations. ExPRES has been developed and used to simulate radio sources of Jupiter [Hess et al., 2008, 2010; Cecconi et al., 2012; Louis et al., 2017], Saturn [Lamy et al., 2008b, 2013], and exoplanets [Hess and Zarka, 2011] and is explained in more details in section 2 of Hess et al. [2008].

In practice, to set up an ExPRES simulation, we define (1) the magnetic and plasma environment around the planet and choose the spatial distribution of point radio sources, (2) their emission angle $\theta$ and the hollow cone thickness $\Delta \theta$ and (3) the location of the observer.

Hereafter, we use the Jupiter magnetic field model ISaAC (In Situ and Auroral Constrain [Hess et al., 2017]), an updated version of the VIPAL model [Hess et al., 2011a] further constrained by the locus of Europa and Ganymede UV auroral footprints, hence particularly adapted to our study. We additionally use the current sheet model of Connerney et al. [1981]. We define the magnetospheric plasma density by $\rho=\rho_{\text {iono }}+\rho_{\text {torus }}$ where $\rho_{\text {iono }}$ and $\rho_{\text {torus }}$ are models of plasma density of the ionosphere and the lo torus, respectively. More precisely,

$$
\rho_{\text {iono }}=\rho_{0_{\text {iono }}} e^{\left(-\left(r-r_{\text {iono }}\right) / H_{\text {iono }}\right)}
$$

with $\rho_{0_{\text {iono }}}=350,000 \mathrm{~cm}^{-3}$ the electron density at the ionospheric peak, $r_{\text {iono }}=650 \mathrm{~km}$, with scale height $H_{\text {iono }}=1600 \mathrm{~km}$ [Hinson et al., 1998], and

$$
\rho_{\text {torus }}=\rho_{0_{\text {torus }}} e^{-\sqrt{\left(r-r_{\text {torus }}\right)^{2}+z^{2}} / H_{\text {torus }}}
$$

with $\rho_{0_{\text {torus }}}=2000 \mathrm{~cm}^{-3}$, the density at the center of the torus, at $r_{\text {torus }}=5.91$ Jovian radii $\left(1 \mathrm{R}_{\text {Jupiter }}=\right.$ $71492 \mathrm{~km}$ ), and a vertical scale height $H_{\text {torus }}=1 \mathrm{R}_{\text {Jupiter }}$ [Bagenal, 1994]. 
Within this environment, we simulate radio sources at frequencies $f$ along magnetic field lines intercepting the Galilean satellites, at the altitudes where $f=f_{\text {ce }}$ (with $f_{\text {ce }}=\frac{e B}{2 \pi m}$, where $B$ is the magnetic field strength, $e$ is the elementary charge, and $m$ is the mass of the electron) whenever (1) $h>650 \mathrm{~km}$ above the ionosphere and (2) $f_{p} / f_{\text {ce }}<0.1$ (with $2 \pi f_{\text {pe }}=\sqrt{\frac{n e^{2}}{m \epsilon_{0}}}$ the local electron plasma frequency, where $n$ is the electron density, and $\epsilon_{0}$ is the permittivity of free space), a condition that must be fulfilled by the CMI to occur [Zarka et al., 2001; Hilgers, 1992]. The corresponding spectrum typically ranges from a few hundreds of $\mathrm{kHz}$ to $20-40 \mathrm{MHz}$. The emission angle $\theta$ was derived following Hess et al. [2008] who successfully simulated lo-DAM arcs by using a loss cone electron distribution function with typical electron energies of $0.64 \mathrm{keV}$ in the north and $3 \mathrm{keV}$ in the south through CMI-driven equations. The loss cone-driven CMI allows to define at each altitude the beaming angle $\theta$ that provides maximum amplification [Mottez et al., 2010]. This yields oblique emission with $\theta$ varying with $f$.

Overall, the only differences between the calculation of $\theta$ by Hess et al. [2008] and ours is that we fixed the auroral peak altitude at $650 \mathrm{~km}$ above the (instead of at) one bar level to fix the ionospheric limit of the loss cone, where electrons are lost by collisions and that we used a different magnetic field model (ISaAC instead of VIT4). These differences slightly change the maximum frequency of the emission from a few tenths to a few $\mathrm{MHz}$, depending on the longitudes of the sources. As in Hess et al. [2008], and unlike in Ray and Hess [2008], we did not simulate any refraction effect, suspected to play a nonnegligible role, especially close to the source [Galopeau and Boudjada, 2016]. The refraction effects need to be taken into account for a better spectral simulation. We neglected them at first order. Finally, we set up the observer at either Voyager or Cassini.

The existence of the lo torus is additionally known to affect the propagation of Alfvén waves which sustain the magnetospheric currents produced by the lo-Jupiter interaction [Neubauer, 1980]. As a result of the increased plasma density within the lo torus, the Alfvén speed is decreased thus the Alfvén waves produced at lo need several tens of minutes to exit the torus. Thus, the emitting field line and the instantaneous field line connected to the moon is different, the former leading the latter by the so-called lead angle, causing a longitude difference between the position of lo and the position of the active flux tube. When lo is at the center of the torus, southern and northern emissions are similarly delayed. When lo reaches the northern (respectively southern) edge of the torus, northern (respectively southern) emissions are quasi-instantaneous, whereas the southern (respectively northern) emissions are strongly delayed. This delay is well known for lo [Saur et al., 2004; Hess et al., 2010], thus, we add an automatic lead angle for the simulation of lo-induced radio emissions, based on the study of Hess et al. [2011a]:

$$
\delta=A+B \cos \left(\lambda_{\mathrm{lo}}-202^{\circ}\right)
$$

with $\lambda_{\mathrm{lo}}$ the jovicentric longitude of lo, $A=2.8$ and $B=-3.5$ in the northern hemisphere and $A=4.3$ and $B=3.5$ in the southern one. No lead angle is included for the other satellites as no such model is available yet.

An example of simulations made with the above described parameters is given in Figure 1, for the four Galilean moons: Io, Europa, Ganymede, and Callisto, over a period of 16 days (one Callisto revolution period) from Day of Year (DoY) 001, 2001 to DoY 016, 2001. The northern emissions (RH polarized) are displayed in white and the southern ones (LH polarized) in black.

Figure 1a the arrows indicate whether lo arcs correspond to A, B, C, or D emission, depending on the sense of curvature (VE or $\mathrm{VL}$ ) and the polarization $(\mathrm{RH}$ or $\mathrm{LH})$, as described in section 1. Note that for the same lo phase (i.e., lo-A, lo-B, lo-C, or lo-D), the arc shapes could be slightly different. This is due to the fact that the magnetic field of Jupiter is not dipolar and axisymmetric. Thus, for one lo phase, we could have different lo jovicentric longitudes, therefore different magnetic field lines connected to lo, and different emissions angles $\theta(f)$.

Another consequence of the complex magnetic field of Jupiter is the high-frequency rotational modulation of the arcs. Since Jupiter rotates faster than the moons, the magnetic field in the flux tubes intercepted by the moons experiences perturbation. Thus, $\theta$ varies as a function of the moon jovicentric longitude [Hess et al., 2008], and we see modulations of the moon-induced CMl emissions at the synodic period of Jupiter and the moons.

It is worth noting that lo-A and lo-B (respectively, lo-C and lo-D) arcs are simulated as portions of a single continuous arc corresponding to the passage of the lo flux tube in the observer's field of view [Hess et al., 2012]. This continuity at high frequencies is not observed in practice for near-equatorial observations, with a range of lo phases where no lo-DAM emission is visible [see Marques et al., 2017]. A possibility is that the 


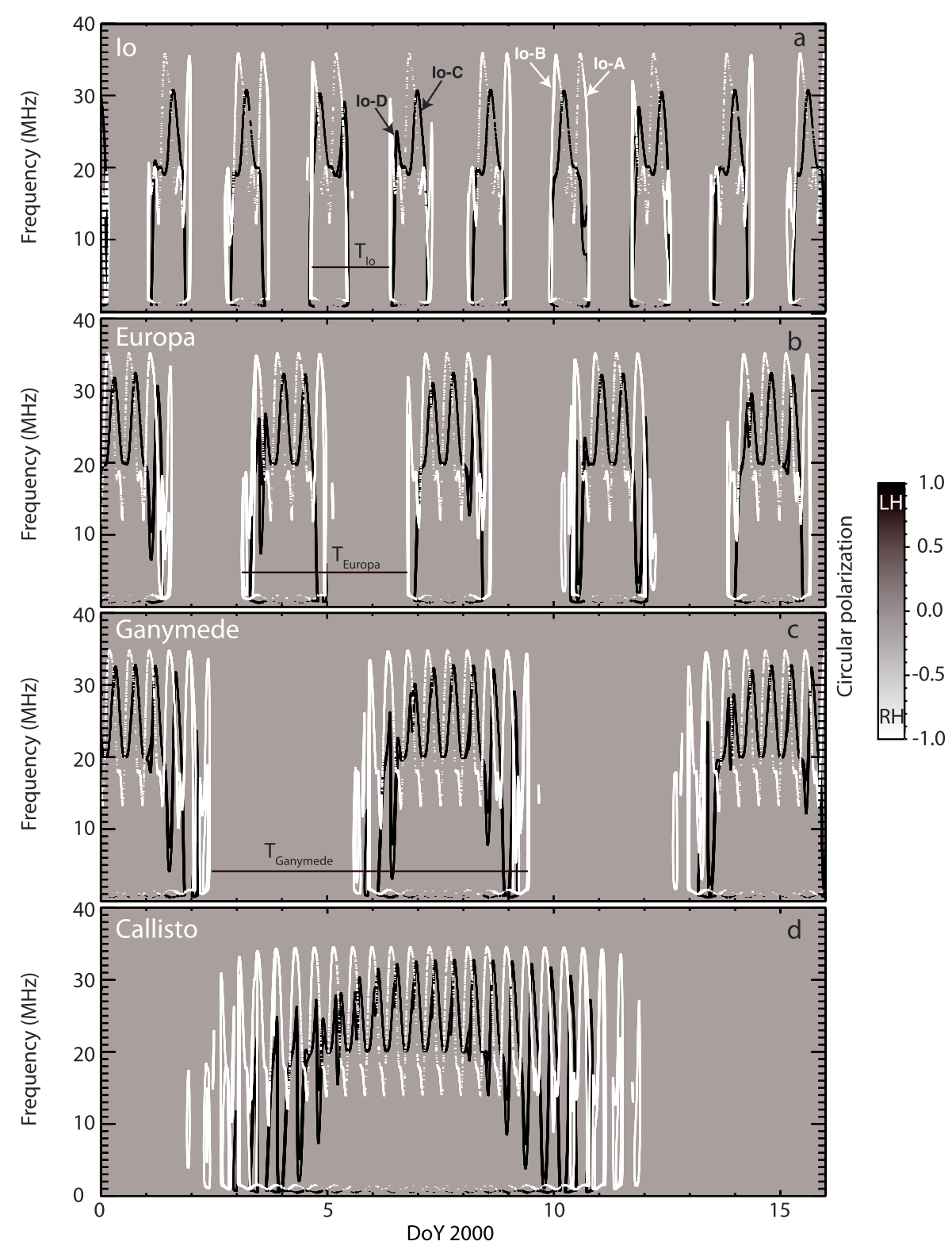

Figure 1. Reference ExPRES simulations from DoY 001, 2000 to DoY 016, 2000 for the Galilean moons (a) lo, (b) Europa, (c) Ganymede, and (d) Callisto. The northern ( $\mathrm{RH}$ ) emissions are represented in white and the southern ones (LH) in black. Sixteen days correspond to one revolution of Callisto. Here the thickness $\Delta \theta$ of the emission cone is deliberately increased (by a factor of 2 ) to improve the visibility of the emission signal.

emission cone is likely oblate, with a reduced value of $\theta$ toward the magnetic equator, making it invisible for near-equatorial observers, or observers in the hemisphere opposed to the source [Galopeau and Boudjada, 2016; Louis et al., 2017].

Figures $1 \mathrm{~b}-1 \mathrm{~d}$ display the simulated dynamic spectrum for Europa-, Ganymede-, and Callisto-induced radio emissions. By analogy with the lo-DAM, we name them Europa-DAM, Ganymede-DAM, and Callisto-DAM emission, since the simulations extend into the DAM range. Furthermore, we will name the VE and VL arcs with the same nomenclature as that employed for lo-DAM emissions [see Marques et al., 2017].

We can see that the duration of a full arc - between the first VE arc and the last VL arc - increases with the orbital period of the moon (increasing from lo to Callisto).

Overall, we made simulations of Europa-, Ganymede-, and Callisto-induced radio emissions across the time interval corresponding to the radio observations of Jupiter obtained by both Voyager during their 
flybys (1979) and Cassini (2000-2003). These spacecraft recorded low-frequency radio observations which are presented in the following section.

\subsection{Observations}

\subsubsection{Voyager}

The Voyager spacecraft had a Planetary Radio Astronomy experiment (PRA) on board connected to two $10 \mathrm{~m}$ long linear wire antennas, sampling the frequency range [1.2 kHz to $40.2 \mathrm{MHz}$, hence, covering the full spectrum of Jupiter auroral radio emissions. The PRA high-resolution ( $6 \mathrm{~s}$ per spectrum) data were extracted from digitized magnetic tapes [Cecconi et al., 2017].

Voyager 1 quasi-continuously observed Jupiter from DoY 001, 1979 to DoY 103, 1979, and flew by the planet on DoY 064, 1979. Voyager 2 observations range from DoY 102, 1979 to DoY 222, 1979, with Jupiter's flyby on DoY 190, 1979.

The Voyager/PRA dynamic spectrum of intensity displayed hereafter was computed similarly to those of Cassini/RPWS described below. To account for the variable spectral response resulting from the antenna resonance, the intensities measured at frequencies beyond $300 \mathrm{kHz}$ were referenced to the $5 \%$ quantile of intensities measured at $289 \mathrm{kHz}$.

\subsubsection{Cassini}

The Cassini mission has the Radio Plasma and Wave Science (RPWS) experiment [Gurnett et al., 2004] on board including five receivers. The high-frequency receiver, connected to three $10 \mathrm{~m}$ electric monopoles, samples the spectral range [3.5 $\mathrm{KHz}$ to $16.125 \mathrm{MHz}$ ], hence, sampling $\mathrm{KOM}, \mathrm{HOM}$, and the low-frequency portion of DAM emissions.

The investigated interval covers the interval from early 2000 to the end of 2003, encompassing the flyby of Jupiter (closest approach on DoY 365, 2000). The high-resolution Cassini dynamic spectrum of flux density and degree of circular polarization displayed in this paper were built after a specific data processing described in Lamy et al. [2008a]. To account for the variable spectral response resulting from the antenna resonance, the intensities measured at frequencies beyond $1500 \mathrm{kHz}$ were additionally referenced to the $10 \%$ quantile of intensities measured at $1475 \mathrm{kHz}$.

\subsection{Detection Criteria for Jupiter Satellite Emissions}

To search for satellite-induced radio emissions, we visually inspected every Voyager/PRA and Cassini/RPWS daily dynamic spectrum over the time intervals specified above and we only retained candidates fulfilling the detection criteria described below.

The observed emission must be an arc with the same curvature as the simulated one (VL or VE), be close enough in time ( $\pm 2 \mathrm{~h}$ for lo, $+2 /-5 \mathrm{~h}$ for Europa, and $+2 /-8 \mathrm{~h}$ for Ganymede, see next paragraph) and at least continuously extend over $3 \mathrm{MHz}$ bandwidth and with a maximum frequency above $5 \mathrm{MHz}$. The emission also needed to be a well-identified single structure (i.e., clearly distinguishable among the other emissions), must not repeat at $9 \mathrm{~h} 55 \mathrm{~min}$ before or after to discard radio sources possibly corotating with Jupiter. Finally, when the polarization was measured (which only concerns Cassini/RPWS observations), the arc polarization had to be consistent with the predicted one.

The time window defined above relies on two sources of uncertainties: the inaccurate modeling of the emission angle $\theta(f)$ and the uncertainty in the lead angle. For lo, the presence of the torus is well known [Bagenal et al., 2014], and we take it into account in our simulations (see equation (3). For Ganymede, Bonfond et al. [2013] noticed multiple spots of the Ganymede UV auroral footprint, a maximum lead angle of $\delta_{\mathrm{Ga}_{\max }}=13^{\circ}$, corresponding to a maximum delay of $\sim-6 \mathrm{~h} 15 \mathrm{~min}$. The negative delay comes from the fact that the lead angle is positive, which means that the emitting flux tube leads the one connected to the moon. In the absence of any similar study for Europa, we used a maximum lead angle of $\delta_{\mathrm{Eu}}=10^{\circ}$ based on a scaling law fitting the extremal lead angles of lo and Ganymede. This $\delta_{\mathrm{Eu}_{\max }}$ corresponds to a maximum delay of $\sim-2 \mathrm{~h} 20 \mathrm{~min}$.

\section{Results}

\subsection{Europa}

Figure 2a shows simulations of lo and Europa emissions from DoY 024, 2001 at 05:00 to DoY 025, 2001 at 05:00. Figures $2 \mathrm{~b}$ and $2 \mathrm{c}$ display Cassini/RPWS dynamic spectrum of flux density and degree of circular polarization over the same time interval. The comparison of these panels reveals that the intense, LH-polarized, 


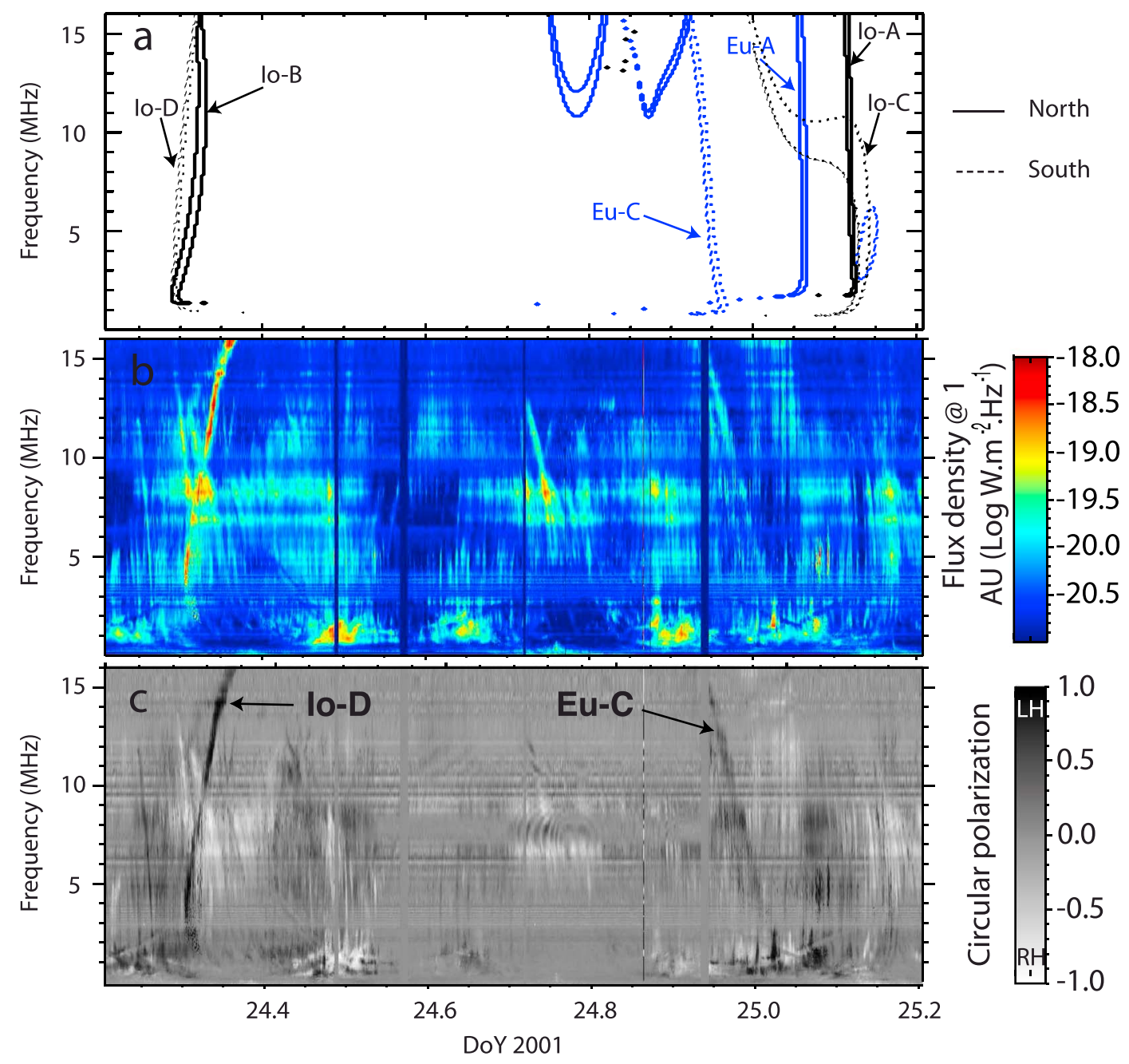

Figure 2. Cassini observations of an lo and a Europa arc. (a) ExPRES simulations (lo in black and Europa in blue - thick line, northern emissions; dotted line, southern emissions), (b) Intensity flux and (c) the circular polarization - LH: left handed (emission from the southern hemisphere); RH: right handed (emission from the northern hemisphere). This time interval was chosen because of the presence of an lo arc and a Europa arc.

VE arc observed in the early portion of the interval strikingly fits the lo-D simulated arc and can therefore be unambiguously attributed to lo. Similarly, the intense, LH-polarized, VL arc observed in the last portion of the interval fits the Europa- $\mathrm{C}$ along the detection criteria presented above, thus we attribute it to Europa.

The Europa arc extends in frequency from $1 \mathrm{MHz}$ to $16 \mathrm{MHz}$, as expected from the simulation. Its shape is similar to that of the lo-D arc, but its intensity is lower by 1 order of magnitude.

The inspection of Cassini/RPWS observations over 2000-2003 revealed 108 Europa events (23 Europa-A, 7 Europa-B, 29 Europa-C, and 49 Europa-D).

Figure 3 shows a second example of a Europa-DAM arc on DoY 055, 1979. This figure shows the quasi-simultaneous observations of Jupiter from both Voyager spacecraft (Figure $3 \mathrm{~b}$ and $3 \mathrm{c}$ display Voyager dynamic spectrum of flux density) together with ExPRES simulations (Figures 3a and 3d). The intensity scale displays a common maximum but a different minimum value to account for different sensitivities.

Voyager 1 was closer to Jupiter $\left(\sim 150 R_{\jmath}\right)$ than Voyager $2\left(\sim 1360 R_{\jmath}\right)$, corresponding to a difference in light travel time of $\sim 5 \mathrm{~min}$. This implies a longitude correction of $\sim 3^{\circ}$ to be considered when comparing the observations. Voyager 1 was at a longitude smaller by $\sim 5^{\circ}$ than Voyager 2 , inducing a delay of $\frac{\Delta \lambda}{\omega}$, with $\omega$ the angular speed of the source of the emissions (either Jupiter or a moon), and $\Delta \lambda$ the difference of longitude between 


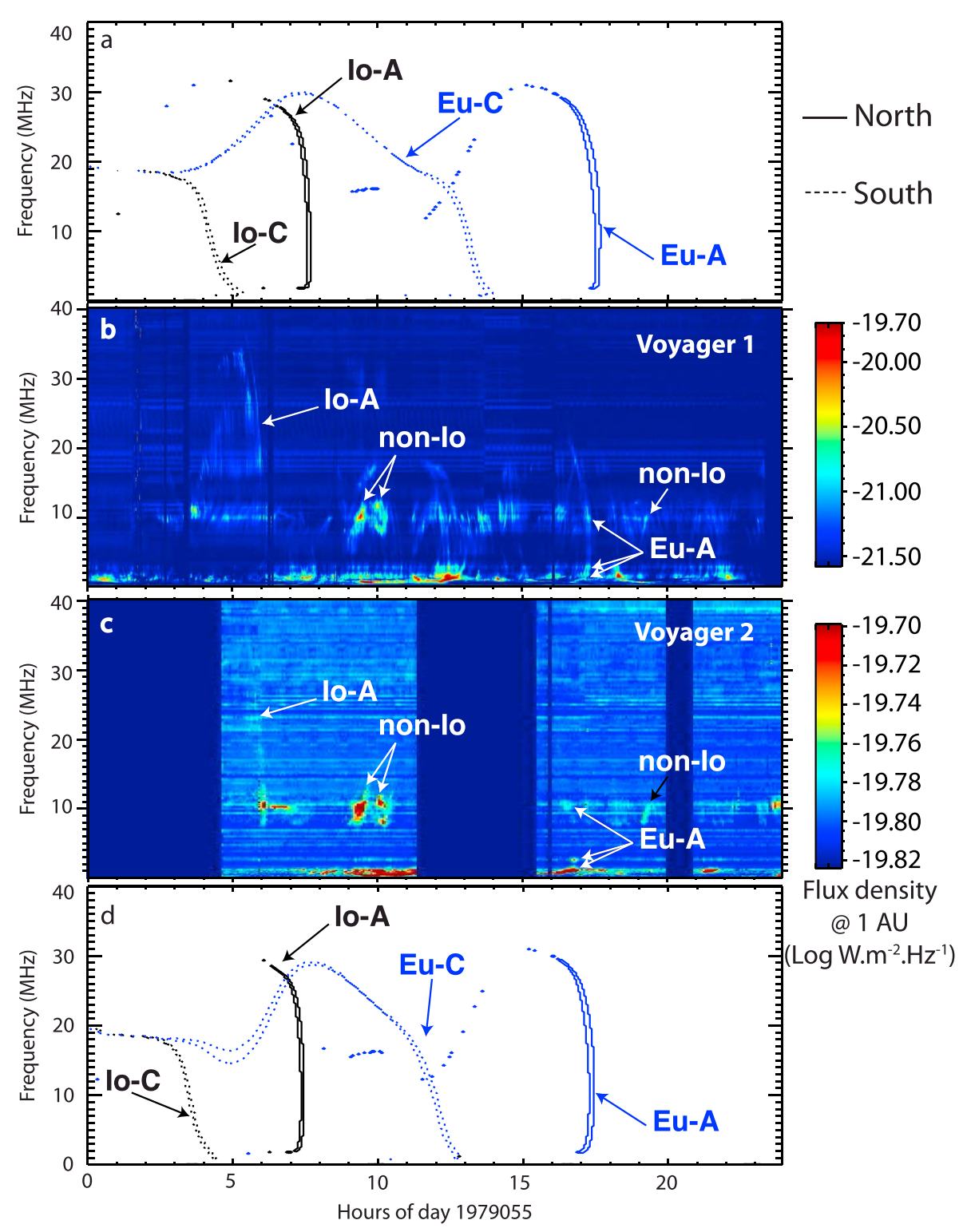

Figure 3. Voyager observations of a Europa and an lo arcs on the DoY 055, 1955. Simulations for (a) Voyager 1 and (d) Voyager 2 (lo in black and Europa in blue - thick line, northern emissions; dotted line, southern emissions). Observations for (b) Voyager 1 and (c) Voyager 2. Voyager 1 was closer to the planet than Voyager 2 and at a longitude larger than Voyager 2. This time interval was chosen because of the presence of an lo arc and a Europa arc.

the observers (corrected for the travel time of the light). Thus, if the emission is stationary over tens of minutes, we are able to determine if the radio sources are corotating with either Jupiter or a moon.

Figure 3b shows Voyager 1 observation of two VL arcs between 05:00 and 06:00 and between 17:00 and 17:30. According to the simulations shown in Figure $3 \mathrm{a}$, we identify these as lo-A and Europa-A emissions.

The Europa arc extends in frequency from $0.5 \mathrm{MHz}$ to $22 \mathrm{MHz}$. The shape is similar to the lo-arc shape, but extends less in frequency than in the case of lo (from $0.5 \mathrm{MHz}$ to $36.5 \mathrm{MHz}$ ).

Figure 3c shows a VL arc between 05:00 and 06:00 consistent with the lo-A simulation (Figure 3d). The time delay between the arc observed by Voyager 1 and the one observed by Voyager 2 is approximately 15 min as expected. The comparison between Figures $3 \mathrm{~b}$ and $3 \mathrm{c}$ also shows that the non-lo emissions (emissions unrelated to satellites) are quasi-simultaneous. We can also see in Figure $3 c$ portions of a single arc between 16:00 and 17:00, close to the simulated Europa-A arc. Figures $4 \mathrm{a}$ and $4 \mathrm{~b}$ show the same observations zoomed 

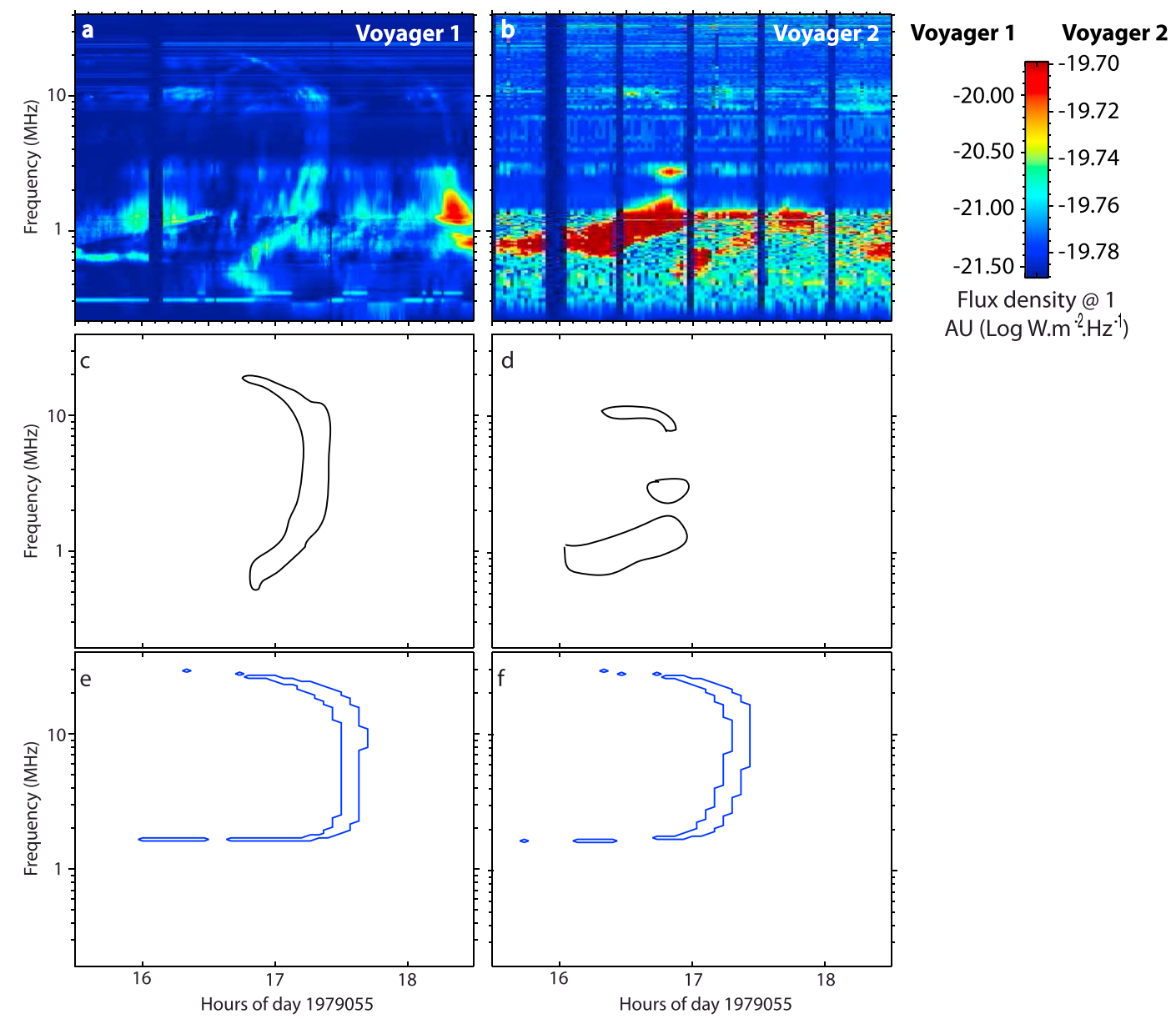

Flux density@ 1 $\mathrm{AU}\left(\log \mathrm{W} \cdot \mathrm{m}^{-2} \cdot \mathrm{Hz} \mathrm{z}^{-1}\right)$

Figure 4. Voyager observations of a Europa arc on the DoY 055, 1955. The left panels ( $a, c$, and e) correspond to Voyager 1 and the right panels ( $b, d$, and $f$ ) to Voyager 2. Figures $4 a$ and $4 b$ are observations, Figures $4 c$ and $4 d$ are contours of the emissions attributed to Europa and Figures $4 \mathrm{e}$ and $4 \mathrm{f}$ are simulations of Europa-induced emissions. Voyager 1 was closer to the planet than Voyager 2, and at a longitude larger than Voyager 2. This figure is a zoom-in version of Figure 3 between $15: 30$ and 18:30.

into the time interval between 15:30 and 18:30 of the observations showed in Figures $3 \mathrm{~b}$ and $3 \mathrm{c}$. Figures $4 \mathrm{c}$ and $4 \mathrm{~d}$ are the contours of the emissions we attributed to Europa, and Figures $4 \mathrm{e}$ and $4 \mathrm{f}$ are the simulations of Europa-C. The time delay between Voyager 1 and Voyager 2 observations of these emissions is approximately 30 mins as expected for an emission induced by Europa.

Note that the emissions measured by Voyager 2 are less intense than those measured by Voyager 1, probably because they have varied between the detection by the two spacecraft.

The comparison between observations and simulations allows us to detect 22 Europa events (6 Europa-A, 9 Europa-B, 2 Europa-C, and 5 Europa-D) for the Voyager 1 and Voyager 2 intervals. The multipoint observations allow us to confirm single-point detections (Figures 3 and 4), but only the detection presented above has been detected with both Voyager spacecraft.

\subsection{Ganymede}

Figure 5a shows a simulation of lo and Ganymede-DAM arcs from DoY 321, 2000 at 17:00 to DoY 322, 2000 at 17:00. Figures $5 b$ and $5 c$ display Cassini/RPWS dynamic spectrum of flux density and degree of circular polarization over the same time interval. The comparison of these three panels reveals that the intense, LH-polarized, VE arc observed between DoY 322.3, 2000 and DoY 322.4, 2000 fits the lo-D simulated arc and can therefore be unambiguously attributed to lo. Similarly, the intense, LH-polarized, VE arc observed between DoY 321.9, 2000 and DoY 322.05, 2000 fits the Ganymede-D simulated arc and can therefore be confidently 


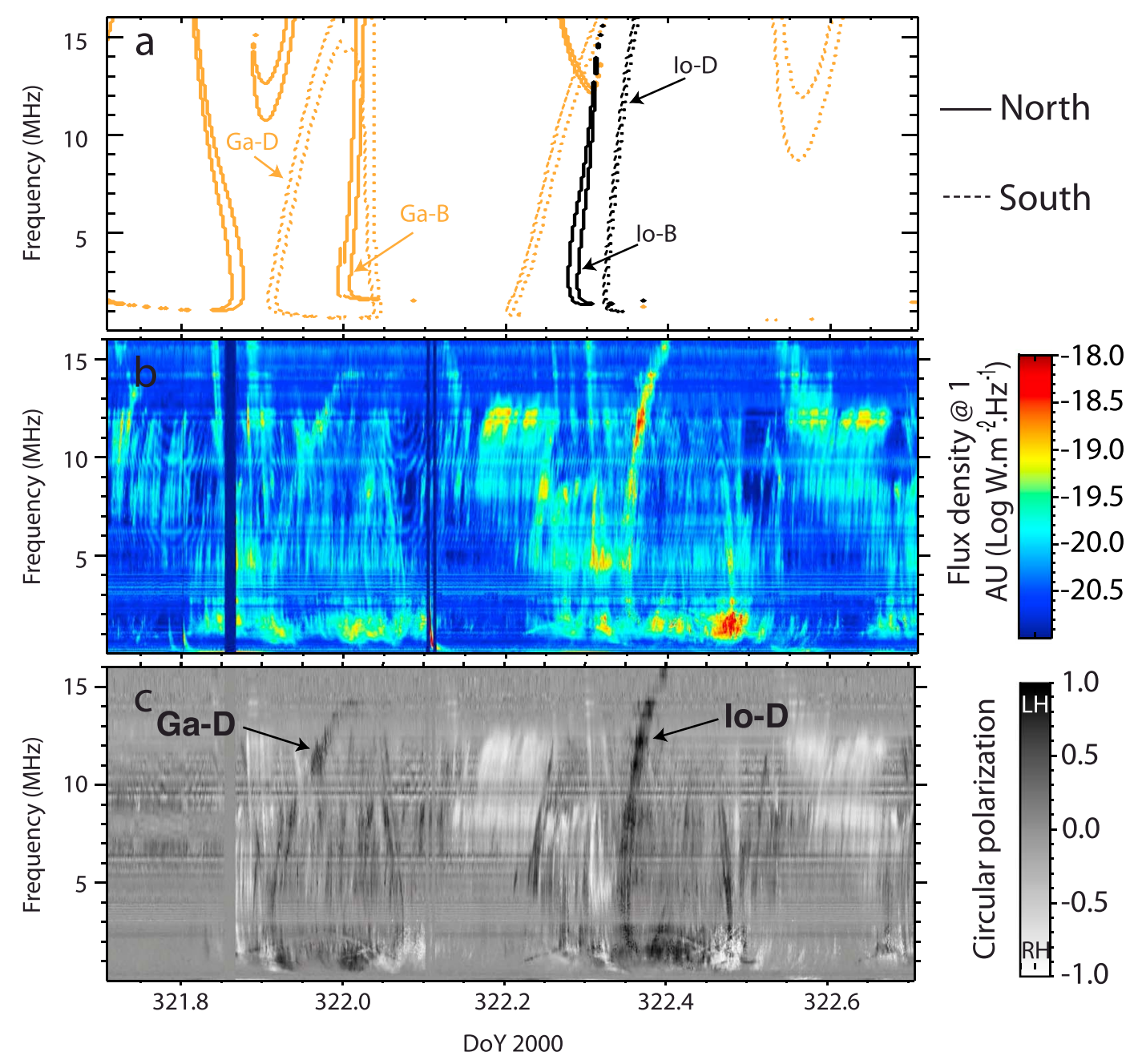

Figure 5. Cassini observations of an lo and a Ganymede arc. (a) ExPRES simulations (lo in black and Ganymede in orange - thick line, northern emissions; dotted line, southern emissions), (b) intensity flux and (c) the circular polarization - LH, left handed (emission from the southern hemisphere); $\mathrm{RH}$, right handed (emission from the northern hemisphere). This time interval was chosen because of the presence of an lo arc and a Ganymede arc.

attributed to Ganymede. The Ganymede arc is extended in frequency, from $0.5 \mathrm{MHz}$ to $14.5 \mathrm{MHz}$. The shape is similar to the lo-D arc shape, but the maximum frequency is lower, and the intensity is lower by 1 order of magnitude.

For the Voyager interval we detected 17 Ganymede events (8 Ganymede-A, 5 Ganymede-B, 1 Ganymede-C, and 3 Ganymede-D). For the Cassini interval we detected 79 Ganymede events (13 Ganymede-A, 13 Ganymede-B, 21 Ganymede-C, and 32 Ganymede-D).

\subsection{Callisto}

Our investigation of 6 months of both Voyager spacecraft data and 4 years of Cassini data did not allow us to detect unambiguous signatures related to Callisto-DAM emissions.

The reasons of no detection could be multiple: (1) The estimated radio power of Callisto is at least 2 orders of magnitude below lo, and one below Europa and Ganymede [Kurth et al., 2000; Zarka, 2007], making it harder to detect; (2) no UV footprint for Callisto has yet definitely been found, suggesting that if the Callisto emissions exist, they are very weak; (3) the revolution of Callisto is very long ( $\simeq 16$ days), thus the uncertainty in the time window is very large. Moreover, if we only look for the VE and VL arcs, considering that we only see the emissions coming from the hemisphere of the spacecraft, the number of detectable events will be $\simeq 18$. 
Table 1. Measured Characteristics of Europa DAM Emission

\begin{tabular}{|c|c|c|c|c|}
\hline $\begin{array}{l}\text { Europa } \\
\text { Component }\end{array}$ & Number & $\begin{array}{c}\text { Min. Frequency (MHz) } \\
\text { Med. } \pm \sigma \\
{[\text { Min-Max] }}\end{array}$ & $\begin{array}{c}\text { Max. Frequency }(\mathrm{MHz}) \\
\text { Med. } \pm \sigma \\
{[\text { Min-Max }]}\end{array}$ & $\begin{array}{c}\text { Duration (min) } \\
\text { Med. } \pm \sigma \\
\text { [Min-Max] }\end{array}$ \\
\hline \multicolumn{5}{|l|}{ Voyager } \\
\hline Europa-A & 6 & $\begin{array}{c}7.0 \pm 5.0 \\
{[0.3-12.0]}\end{array}$ & $\begin{array}{c}22.0 \pm 8.5 \\
{[12.0-34.0]}\end{array}$ & $\begin{array}{l}35.0 \pm 18.1 \\
{[15.0-60.0]}\end{array}$ \\
\hline Europa-B & 9 & $\begin{array}{l}14.0 \pm 6.8 \\
{[0.8-21.0]}\end{array}$ & $\begin{array}{c}21.0 \pm 7.1 \\
{[11.0-31.0]}\end{array}$ & $\begin{array}{c}35.0 \pm 45.9 \\
{[30.0-175.0]}\end{array}$ \\
\hline Europa-C & 2 & $\begin{array}{c}- \\
{[0.7-1.0]}\end{array}$ & $\begin{array}{c}- \\
{[13.0-13.5]}\end{array}$ & $\begin{array}{c}- \\
{[55.0-100.0]}\end{array}$ \\
\hline Europa-D & 5 & $\begin{array}{c}7.5 \pm 3.1 \\
{[6.0-13.0]}\end{array}$ & $\begin{array}{c}12.0 \pm 4.6 \\
{[10.5-21.0]}\end{array}$ & $\begin{array}{c}50.0 \pm 81.3 \\
{[15.0-215.0]}\end{array}$ \\
\hline Cassini & & & & \\
\hline Europa-A & 23 & $\begin{array}{c}3.0 \pm 3.1 \\
{[0.5-12.0]}\end{array}$ & $\begin{array}{l}16.0 \pm 2.4 \\
{[6.0-16.0]}\end{array}$ & $\begin{array}{l}40.0 \pm 12.3 \\
{[20.0-80.0]}\end{array}$ \\
\hline Europa-B & 7 & $\begin{array}{l}2.0 \pm 2.5 \\
{[1.0-8.0]}\end{array}$ & $\begin{array}{l}16.0 \pm 3.6 \\
{[6.5-16.0]}\end{array}$ & $\begin{array}{l}40.0 \pm 11.7 \\
{[30.0-60.0]}\end{array}$ \\
\hline Europa-C & 29 & $\begin{array}{l}2.0 \pm 2.0 \\
{[0.5-7.5]}\end{array}$ & $\begin{array}{c}13.0 \pm 2.8 \\
{[7.25-16.0]}\end{array}$ & $\begin{array}{c}40.0 \pm 23.3 \\
{[20.0-105.0]}\end{array}$ \\
\hline Europa-D & 49 & $\begin{array}{l}2.0 \pm 1.9 \\
{[0.5-8.0]}\end{array}$ & $\begin{array}{l}12.0 \pm 2.6 \\
{[6.0-16.0]}\end{array}$ & $\begin{array}{c}60.0 \pm 46.5 \\
{[15.0-285.0]}\end{array}$ \\
\hline
\end{tabular}

\section{Properties of Jupiter Satellite Emissions}

\subsection{Spectral and Temporal Characteristics}

The first consequence of the detection of 130 Europa-DAM emissions and 96 Ganymede-DAM emissions is that we can now describe the time duration and frequency characteristics of each component of these emissions. Tables 1 and 2 give all the median and extreme values of frequencies and time duration for each Europa-DAM and Ganymede-DAM component, respectively.

The maximum frequency provided by the Cassini detections is biased, because the upper frequency limit of Cassini/RPWS is restricted to $16 \mathrm{MHz}$. However, the Cassini detections reached the $16 \mathrm{MHz}$ value in $\sim 27 \%$ of the Europa components and $\sim 20 \%$ of the Ganymede components only. Thus, the median (rather than the mean) value of $f_{\text {max }}$ remains reliable.

For Europa and Ganymede $C$ and $D$ components, the median maximum frequency of Voyager detections remains below $16 \mathrm{MHz}$, as for Cassini. As a result, we used the whole set of Cassini and Voyager detections to assess a median value of $f_{\max }$ with more statistical weight.

As the observed maximum frequencies of most of the Europa and Ganymede $A$ and $B$ components are higher - and above $16 \mathrm{MHz}$ - for Voyager than for Cassini (truncated at $16 \mathrm{MHz}$ ), we consider the Voyager median maximum frequency more reliable for these two components.

Since the median minimum frequencies of the emissions seen by Cassini are within 1-3 MHz, while the ones of the emissions seen by Voyager are within 5-10, we choose to use the median minimum frequencies of the emissions seen by Cassini, which seems to be more sensitive at low frequencies.

For the average time duration we choose to use the median for all the detections, and give a lower limit of the median duration of the emissions.

Table 3 gives a summary of the median frequency range and the median duration of each Europa-DAM and Ganymede-DAM component.

Since the frequency range of the observed Europa and Ganymede emissions extends into the DAM range, our proposition to name them Europa-DAM and Ganymede-DAM, by analogy with the lo-DAM emission, is therefore fair. 
Table 2. Measured Characteristics of Ganymede DAM Emission

\begin{tabular}{|c|c|c|c|c|}
\hline $\begin{array}{l}\text { Ganymede } \\
\text { Component }\end{array}$ & Number & $\begin{array}{c}\text { Min. Frequency }(\mathrm{MHz}) \\
\text { Med. } \pm \sigma \\
{[\text { Min-Max] }}\end{array}$ & $\begin{array}{c}\text { Max. Frequency }(\mathrm{MHz}) \\
\text { Med. } \pm \sigma \\
{[\text { Min-Max }]}\end{array}$ & $\begin{array}{l}\text { Duration (min) } \\
\text { Med. } \pm \sigma \\
\text { [Min-Max] }\end{array}$ \\
\hline \multicolumn{5}{|l|}{ Voyager } \\
\hline Ganymede-A & 8 & $\begin{array}{c}8.0 \pm 5.0 \\
{[1.0-15.0]}\end{array}$ & $\begin{array}{c}23.0 \pm 7.2 \\
{[12.0-32.0]}\end{array}$ & $\begin{array}{l}30.0 \pm 10.2 \\
{[10.0-39.0]}\end{array}$ \\
\hline Ganymede-B & 5 & $\begin{array}{l}16.0 \pm 4.8 \\
{[7.0-19.0]}\end{array}$ & $\begin{array}{c}28.0 \pm 6.9 \\
{[20.0-38.0]}\end{array}$ & $\begin{array}{l}30.0 \pm 16.8 \\
{[10.0-50.0]}\end{array}$ \\
\hline Ganymede-C & 1 & $\begin{array}{c}- \\
{[1.0]}\end{array}$ & $\begin{array}{c}- \\
{[12.0]}\end{array}$ & $\begin{array}{c}- \\
{[20.0]}\end{array}$ \\
\hline Ganymede-D & 3 & $\begin{array}{l}7.0 \pm 0.0 \\
{[7.0-7.0]}\end{array}$ & $\begin{array}{c}11.5 \pm 0.5 \\
{[11.0-12.0]}\end{array}$ & $\begin{array}{l}60.0 \pm 16.1 \\
{[35.0-65.0]}\end{array}$ \\
\hline Cassini & & & & \\
\hline Ganymede-A & 13 & $\begin{array}{l}2.0 \pm 1.9 \\
{[0.5-6.5]}\end{array}$ & $\begin{array}{l}14.0 \pm 2.9 \\
{[7.0-16.0]}\end{array}$ & $\begin{array}{l}40.0 \pm 20.9 \\
{[20.0-95.0]}\end{array}$ \\
\hline Ganymede-B & 13 & $\begin{array}{l}1.0 \pm 1.9 \\
{[0.5-7.5]}\end{array}$ & $\begin{array}{l}14.0 \pm 2.6 \\
{[7.0-16.0]}\end{array}$ & $\begin{array}{c}50.0 \pm 25.3 \\
{[20.0-100.0]}\end{array}$ \\
\hline Ganymede-C & 21 & $\begin{array}{l}1.0 \pm 1.8 \\
{[0.5-8.0]}\end{array}$ & $\begin{array}{l}13.0 \pm 2.9 \\
{[7.0-16.0]}\end{array}$ & $\begin{array}{c}60.0 \pm 21.3 \\
{[15.0-100.0]}\end{array}$ \\
\hline Ganymede-D & 32 & $\begin{array}{c}2.0 \pm 2.6 \\
{[0.25-10.0]}\end{array}$ & $\begin{array}{r}12.5 \pm 2.2 \\
{[7.0-16.0]}\end{array}$ & $\begin{array}{c}60.0 \pm 40.5 \\
{[20.0-210.0]}\end{array}$ \\
\hline
\end{tabular}

\subsection{Occurrence}

Figure 6 displays the Phase-CML distributions of detected events separately for Europa (Figures $6 a-6 c$ ) and Ganymede (Figures $6 \mathrm{~d}-6 \mathrm{f}$ ) for Cassini (Figures $6 \mathrm{a}$ and $6 \mathrm{~d}$ ), and Voyager (Figures $6 \mathrm{~b}$ and $6 \mathrm{e}$ ) observations, and for all observations (Figures $6 \mathrm{c}$ and $6 \mathrm{f}$ ). Each line segment corresponds to a detected emission. The northern emissions (i.e., RH polarized) are represented in blue and the southern (i.e., LH polarized) in red.

We see that the events are grouped in two regions of phases around $100^{\circ}$ and $260^{\circ}$, resulting from our selection criteria, corresponding to the VE and VL part of the arcs. We also see that the emissions are grouped in $\mathrm{CML}$, unlike the distribution of simulated emissions (see Appendix A, Figure A1), which is not produced by our selection criteria.

The Voyager and Cassini observations show similar results, and combining both of them (see Figures $6 c$ and $6 f$ ) allows us to define regions of maximum occurrence for each Europa-DAM and Ganymede-DAM component.

Table 3. Median Characteristics of Europa-DAM and Ganymede-DAM Emissions

\begin{tabular}{lcccc} 
Component & Number & $\begin{array}{c}\text { Minimum } \\
\text { Frequency }(\mathrm{MHz})\end{array}$ & $\begin{array}{c}\text { Maximum } \\
\text { Frequency }(\mathrm{MHz}) \\
\text { (Maximum Extent) }\end{array}$ & $\begin{array}{c}\text { Duration } \\
(\mathrm{min})\end{array}$ \\
\hline $\begin{array}{l}\text { Europa-DAM } \\
\text { Europa-A }\end{array}$ & 29 & 3.0 & $22.0(\geq 30)$ & $40-45$ \\
Europa-B & 16 & 2.0 & $22.0(\geq 30)$ & $40-45$ \\
Europa-C & 31 & 2.0 & $13.0(\geq 16)$ & $50-70$ \\
Europa-D & 54 & 2.0 & $13.0(\geq 16)$ & $50-70$ \\
Ganymede-DAM & & & & \\
Ganymede-A & 21 & 2.0 & $22.0(\geq 30)$ & $\sim 40$ \\
Ganymede-B & 18 & 1.0 & $22.0(\geq 30)$ & $\sim 40$ \\
Ganymede-C & 22 & 1.0 & $13.0(\geq 16)$ & $\sim 60$ \\
Ganymede-D & 35 & 2.0 & $13.0(\geq 16)$ & $\sim 60$ \\
\hline
\end{tabular}



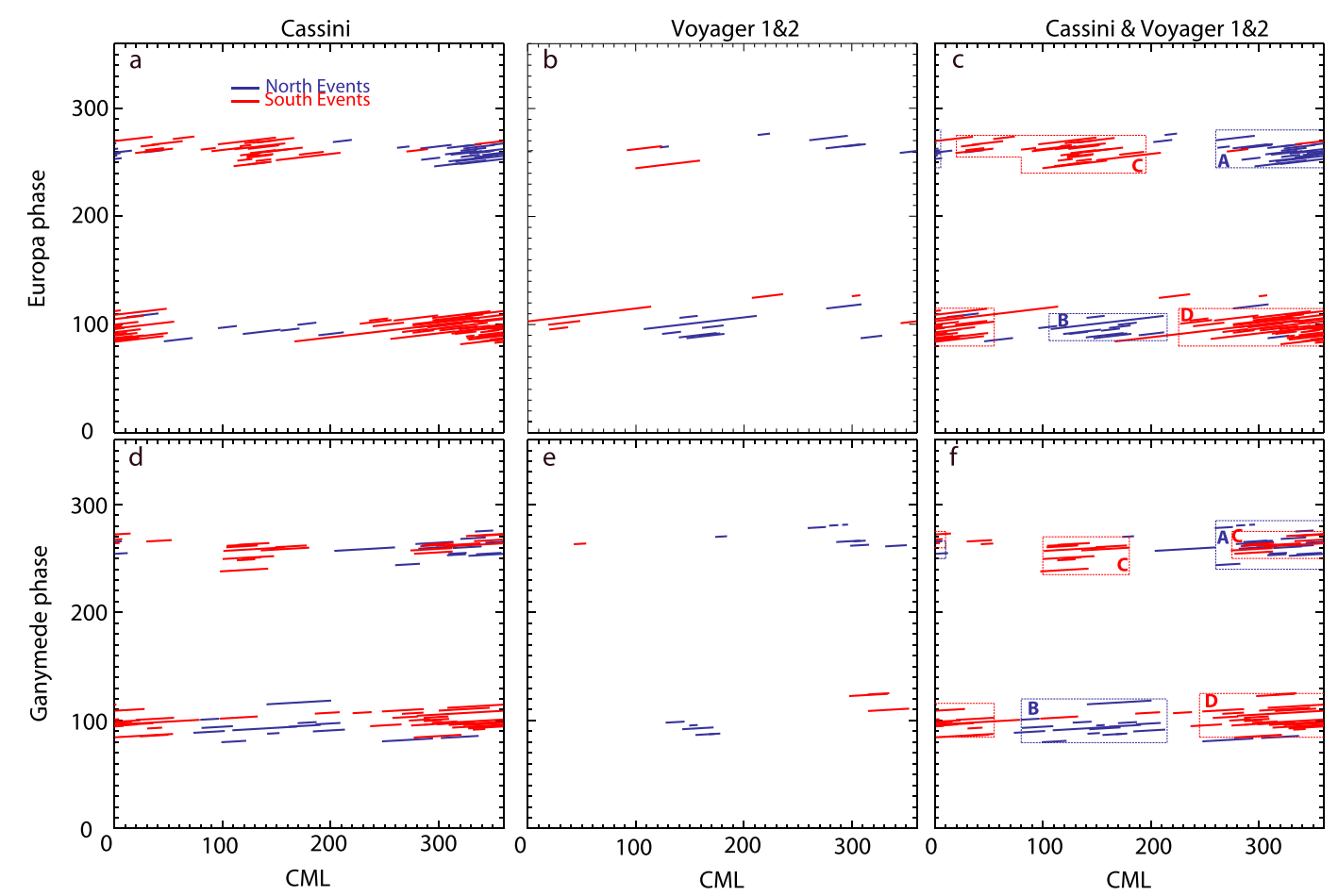

Figure 6. Satellite phase versus observer CML diagrams of ( $a, b$, and c) Europa- and ( $d$, e, and f) Ganymede-detected events seen by Cassini (Figures $6 \mathrm{a}$ and $6 \mathrm{~d}$ ) and the Voyager spacecraft (Figures $6 \mathrm{~b}$ and $6 \mathrm{e}$ ). Figures $6 \mathrm{c}$ and $6 \mathrm{f}$ contain all the detections. The northern events are represented in blue and the southern ones in red.

The regions are summarized in Table 4 for Europa and Table 5 for Ganymede, and plotted in Figures $6 \mathrm{c}$ and $6 \mathrm{f}$ with boxes. The boxes have been defined with the criteria that there are at least three events in the regions, and from the moment there is a CML recovery of two emissions. The regions have a precision of $5^{\circ}$ in phase and CML.

Figure 7 displays the histogram (in bin of $5^{\circ}$ ) of the cumulative duration of all the events as a function of the jovicentric longitude of the Galilean moons. The northern emissions are plotted in blue and the southern ones in red. Figure 7a displays the histogram of Europa-DAM emissions and Figure 7b the histogram of the Ganymede-DAM emissions. For Europa-DAM this distribution shows discontinuities: the occurrence varies significantly as a function of the moon jovicentric longitude and peaks around $\sim 250^{\circ}$ for the north and around $\sim 60^{\circ}$ for the south. The distribution for Ganymede-DAM shows the same, with a second enhancement near $245^{\circ}$ for the southern emissions. This figure suggests that not all the Europa/Ganymede jovicentric longitudes are active, and it seems that there are preferential longitudes, as for lo-DAM emissions [see Marques et al., 2017]. The range of active longitudes for each component is described in Tables 4 and 5.

\subsection{Temporal Variability}

Figures $8 \mathrm{a}$ and $8 \mathrm{~b}$ show the occurrence of the detected Europa-DAM and Ganymede-DAM events as a function of time. It is binned in intervals of 30 days. Figure 8 a deals with Voyager 1 and 2 observations and Figure $8 \mathrm{~b}$ for

Table 4. CML and Europa Phase ( $\phi_{\text {Europa }}$ ) Boundaries of Jupiter DAM Emissions Controlled by
Europa (cf. Figure 6) and Corresponding Active Jovicentric Longitude $\left(\lambda_{\text {Europa }}\right)$ (cf. Figure 7a)
\begin{tabular}{lcccc} 
Europa Component & CML (deg) & $\phi_{\text {Europa }}($ deg) & $\lambda_{\text {Europa }}(\mathrm{deg})$ & Number of Detections \\
\hline A & $260-5$ & $245-280$ & $180-295$ & 29 \\
B & $105-215$ & $85-110$ & $190-285$ & 16 \\
C & $20-80$ & $250-275$ & $0-115$ & 31 \\
& $80-195$ & $240-275$ & $250-335$ & \\
D & $225-55$ & $80-115$ & $260-140$ & 54 \\
\hline
\end{tabular}


Table 5. CML and Ganymede Phase ( $\phi_{\text {Ganymede }}$ ) Boundaries of Jupiter DAM Emissions Controlled by Ganymede (cf. Figure 6) and Corresponding Active Jovicentric Longitude ( $\lambda_{\text {Ganymede }}$ ) (cf. Figure 7b)

\begin{tabular}{lcccc} 
Ganymede Component & CML (deg) & $\phi_{\text {Ganymede }}(\mathrm{deg})$ & $\lambda_{\text {Ganymede }}(\mathrm{deg})$ & Number of Detections \\
\hline A & $0-10$ & $250-265$ & $160-285$ & 21 \\
& $255-360$ & $240-285$ & & 18 \\
B & $80-215$ & $80-120$ & $165-290$ & 22 \\
C & $0-10$ & $260-275$ & $25-95$ & \\
& $100-180$ & $235-270$ & & 35 \\
& $275-360$ & $250-275$ & & \\
\hline
\end{tabular}

Cassini ones. The blue dotted line corresponds to the Europa emissions, the red line to the Ganymede emissions and the grey line to the total number of emissions. Figures $8 \mathrm{c}$ and $8 \mathrm{~d}$ show the distance of the spacecraft (in $\mathrm{AU}$ ) to Jupiter as a function of time. Finally, Figures $8 \mathrm{e}$ and $8 \mathrm{f}$ show the magnetic latitude (minimum, mean, and maximum) of the spacecraft as a function of time. The shaded intervals represent, for Voyager, the period of low-resolution mode, and for Cassini the data gaps.

We see (Figures $8 \mathrm{a}$ and $8 \mathrm{~b}$ ) two remarkable variations: (1) the number of events varies with the distance to Jupiter (see Figures $8 \mathrm{c}$ and $8 \mathrm{~d}$ ). Indeed for both Voyager (Figure 8a) the most significant number of events is detected near the flyby, and for Cassini there is a local maximum at the closest approach (Figure $8 \mathrm{~b}$ ). This expected behavior directly results from the increasing sensitivity of radio instruments with decreasing distances to the planet. And (2) the number of events unexpectedly varies with a gap at the end of year 2001 (Figure $8 \mathrm{~b}$ ) and followed by a large increase at the beginning of year 2002 producing a second maximum, the amplitude of which exceeds the first one. Possible reasons for this unexpected variation are discussed in section 5.3 .
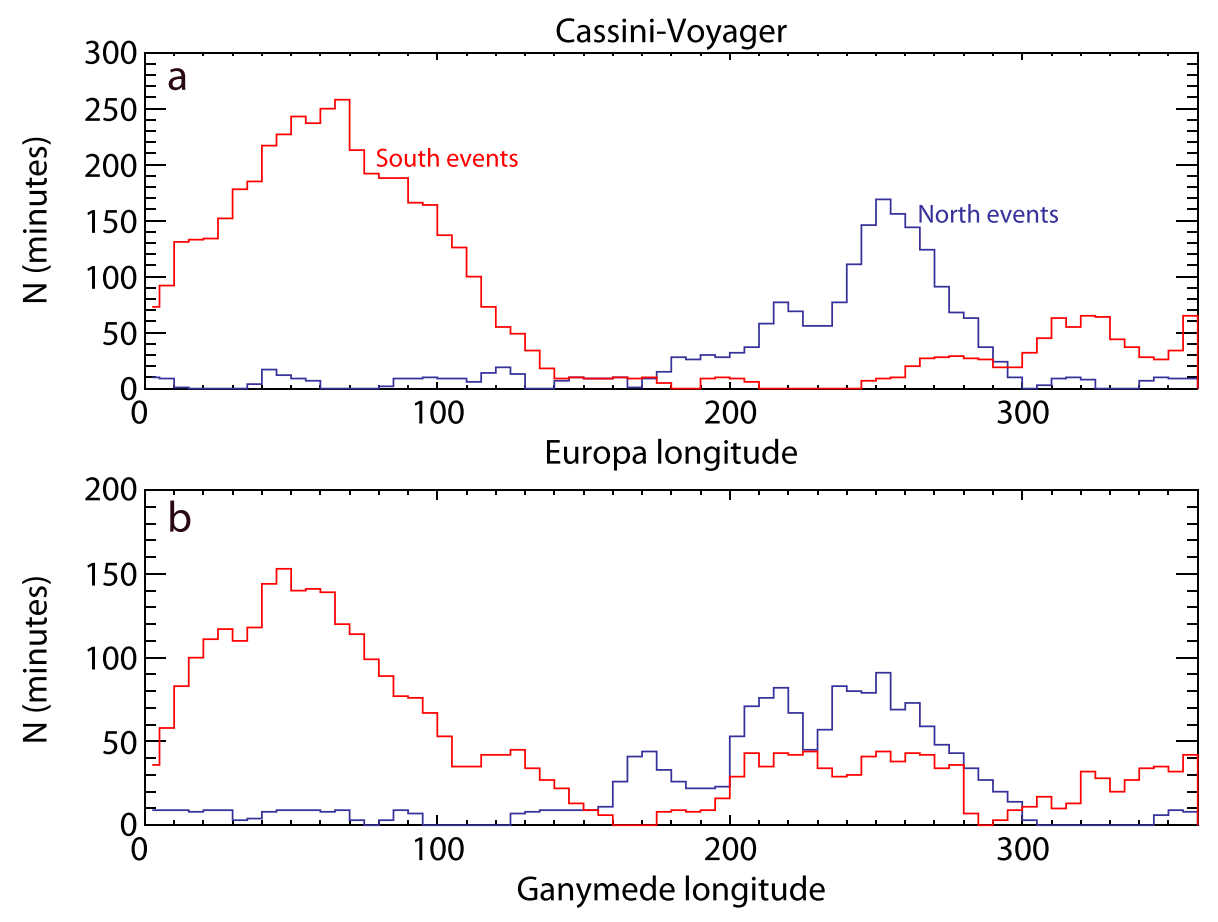

Figure 7. These panels show the histograms (in bin of $5^{\circ}$ ) of the cumulative duration of all the events of (a) Europa- and (b) Ganymede-induced emissions as a function of the jovicentric longitudes of the moons. See Tables 4 and 5 for the details of active longitudes by components. The northern events are represented in blue and the southern ones in red. 

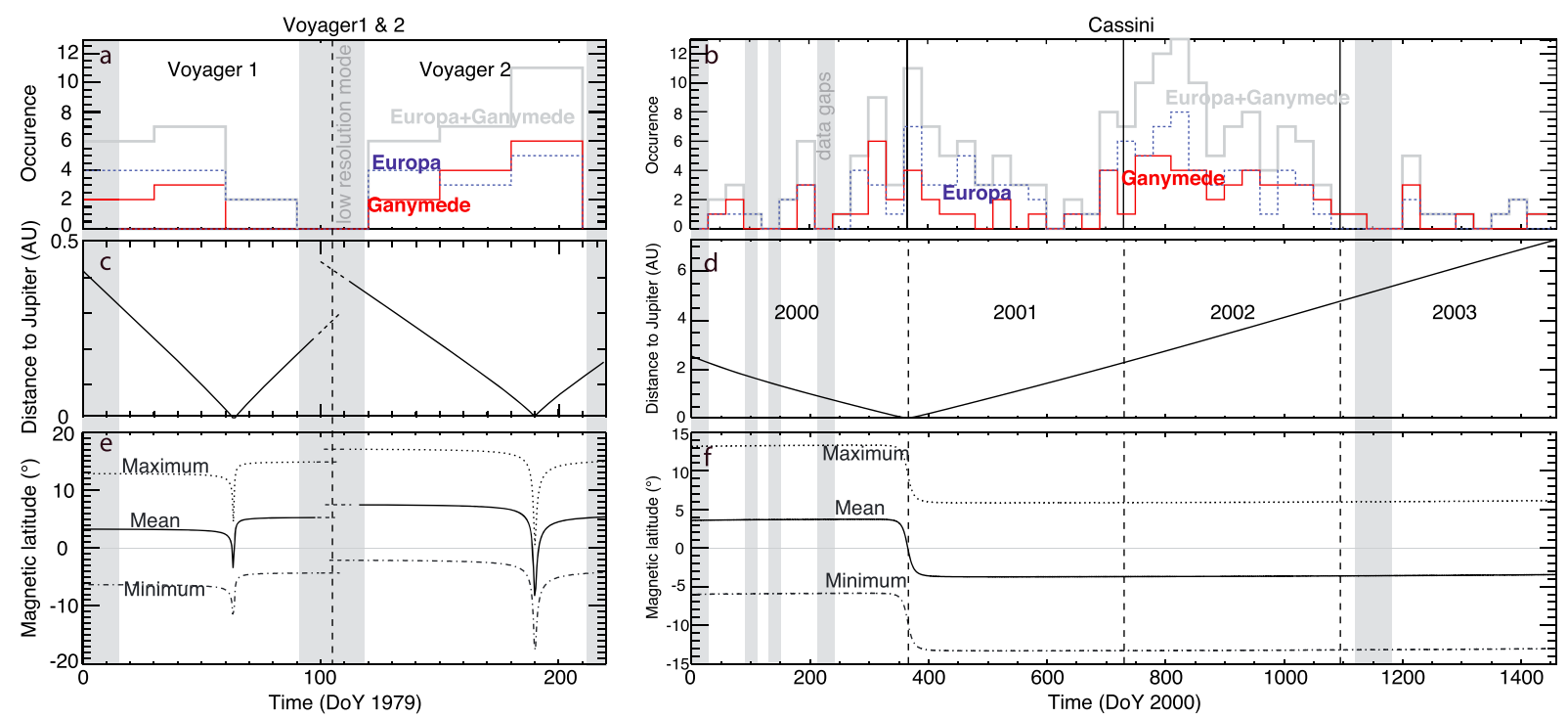

Figure 8. Histograms of detections. ( $a, b)$ The number of detections for Ganymede (red line), Europa (blue dotted line), and both Europa and Ganymede (grey line) with Voyager 1 and 2 (left column) and Cassini (right column) as a function of time (same $y$ axis scale). The distance of the spacecraft to Jupiter (in AU) is plotted in (c) Voyager 1 and 2 and (d) Cassini. The spacecraft magnetic latitude (minimum, mean, and maximum) is plotted in (e) Voyager 1 and 2 and (f) Cassini.

In Figures 8a and 8b we see that the occurrence of Ganymede-DAM and Europa-DAM are correlated. The linear correlation coefficient is equal to 0.65 . We will further discuss this correlation in section 5.3 .

Note that because of the resonance between lo, Europa, and Ganymede (ratio of orbital periods 1:2:4), we have six emissions in our catalog, whose origin (either Europa or Ganymede DAM) can not be unambiguously identified. We did not take them into account in our study. There was no ambiguity with lo because lo-DAM is often 1 order of magnitude more intense.

\section{Discussion}

\subsection{Simulation Parameters}

The fact that the ionospheric limit of the loss cone is taken at the peak altitude of the UV emissions, the addition of two density models (torus and ionospheric), and the use of the new magnetic field model ISaAC [see Hess et al., 2017] allow us to have a better simulation of the lo arcs in terms of time and frequency.

On the other hand, ExPRES is intrinsically limited by the assumptions described in section 2.1. Hess et al. [2008] showed that even in the case of well-known lo-arcs, simulations do not perfectly fit the observations, in terms of arcs shape and curvature, which are controlled by $\theta$. An example of Europa-DAM emission with constant $\theta$ is plotted in Figure B1 of Appendix B. In the case of the loss cone distribution function, the parameters that control the value of $\theta$ are the electron energy, the magnetic field model, and the $f_{\max }$ fixed by the ionosphere altitude [see Hess et al., 2008, Figure 2]. For a given magnetic field line, and a given value of the energy of the electrons, the values of $\theta$ are fixed by frequency and longitudes. Thus, here the principal known limitations are the following: (1) the electron kinetic energy used is the same as that used in the lo case, because we expect the same electrons population for Europa and Ganymede; (2) we used a loss cone electron distribution function through CMI equations, because of the successful previous studies for lo.

Despite these assumptions and uncertainty in the simulations, we know that the simulations match the observation on long time scale, in a time window of $\sim 1-2 \mathrm{~h}$ [see Louis et al., 2017, Figure 1], thus even if the simulations do not perfectly fit the observations, they allow us to constrain a time and frequency location of the radio emissions.

Note that the refraction effects at the source, and along the ray path, may significantly affect the simulations [see Ray and Hess, 2008; Galopeau and Boudjada, 2016]. These effects are not taken into account in these simulations. 


\subsection{Selection Criteria}

Our detections depend on the selection criteria described in section 2.3. Because of the complex morphology of our simulations (see limitation discussed in section 5.1, and the reasons of the visibility of only part of the arcs (see section 2.1)), we chose to focus on the VE and VL portions of a full arc which extend below $20 \mathrm{MHz}$, hence excluding from the analysis the high-frequency oscillations seen in our simulations (Figure 1). This, in turn, reduces a bit the range of satellite phases in Figure 6. Figure A1 in Appendix A shows comparison between the satellite phases versus CML diagrams of the simulated emissions with and without the high-frequency modulations. We see that there are two peaks in the phase distribution, regardless of whether we select only the VE and the VL arcs or all the emissions.

To confirm our detections, we used the polarization, whenever it was available (which depends on the instrumental mode) and usable (the derived wave polarization mainly depends on the antenna orientation with respect to the incoming wave and its signal-to-noise ratio). The polarization was usable for 67 arcs out of 238 . In these 67 events we confirmed 61 and eliminated 6 cases which did not have the expected polarization. This ratio provides a first estimate of the number of possible false detections less than $9 \%$. As this number is low, this does not change the median properties described in section 4.

\subsection{Jupiter Satellite Interactions}

The statistical detection of induced emissions by two Galilean moons besides lo confirm that different types of planet-satellite interactions produce radio emissions. Indeed Ganymede has an intrinsic magnetic field and lo and Europa do not.

In section 4, we demonstrated that there is a linear correlation between the occurrence of Europa and Ganymede events (see Figure 8). This linear correlation of 0.65 shows that the Jupiter-Europa and JupiterGanymede interactions are similarly affected by the magnetospheric conditions. Since the electrons responsible for the emissions are accelerated by the continuity of the current carried along the Alfvén waves, and that it depends on the Alfvén conductance-which depends on the plasma density [see Hess et al., 2011b, and references therein, equations (3) and (4)] — the variation of the detected events could be a sign of the variability of the plasma conditions between 9 and 15 Jovian Radii. Since we do not detect events during an interval of several days at the end of the year 2001 (see Figure 8b), this suggests that the level of activity went below the detection threshold before it increased again in 2002 at unprecedented amplitude.

If Europa-DAM and Ganymede-DAM were emitted all the time, we would expect the moon-planet interaction of Ganymede 50\% stronger than Europa, because Ganymede's revolution is twice as slow as Europa's. Here the ratio between the detected Ganymede-DAM and Europa-DAM is $75 \%$. We can therefore conclude that Ganymede is $\sim 50 \%$ more active than Europa.

The intensity order of magnitude of Europa-DAM and Ganymede-DAM is roughly the same, and we see that the intensity is lower by 1 order of magnitude than lo-DAM. This result agrees with the studies of Zarka [2007] and Hess et al. [2011b].

If we zoom in on Figure 1 at the time where the first arcs appear (respectively last arcs disappear), we see simulated VL (respectively VE) arcs. In practice for lo we never observe such arcs. It could be a wrong estimation of the opening angle $\theta$, but it also could have a physical origin. Indeed we know that the angular speed of each satellite and Jupiter is not very different, but, on the other hand, the fraction of Jupiter's rotation is different for each satellite during the time the satellite is at an observable phase. This fraction of time increases with the augmentation of the time duration of the revolution. For a source in corotation with Jupiter, we observe first a VE arc and then a VL arc few hours later, representing a fraction of the rotation period of Jupiter [see Hess et al., 2014, Figure 2]. The simulations for the Galilean moons show two things: (1) the variation of the active longitudes (modulations) and (2) half the revolution period of the moon (global shape of the simulations over several hours). Indeed, if the magnetic field was axisymmetric, the shape of the emission should be an arc during half the period of the revolution of the moon, without modulations.

At the appearance/disappearance of the moons, this variation of the active longitudes (faster for Europa, Ganymede, and Callisto than for lo) could strongly modulate the observed arc and leads to the observation of VL arcs on the west side and VE arcs on the east side. In our detections we observed a few cases of VL arc on the west side ( 1 Ganymede-B and 1 Ganymede-D) and VE arcs on the east side (1 Europa-C, 1 Ganymede-A, and 6 Ganymede-C) with the same median properties as the other Europa- and Ganymede-induced radio emissions described in section 4. 


\subsection{Comparison With lo-DAM}

The occurrence probability regions of Europa and Ganymede of Figure 6 overlap with each other (with a larger extent in CML for Ganymede-C). The occurrence probability regions of Ganymede-DAM and Europa-DAM have roughly the same boundaries than lo-DAM emissions, with a supplementary extension in CML of the south components compared to lo-DAM emissions [see Marques et al., 2017].

As for lo-DAM, the Europa-DAM and Ganymede-DAM emission are grouped in jovicentric longitude of the moon (Figure 7). Unlike the lo-DAM seen by Marques et al. [2017], the Europa-DAM and Ganymede-DAM southern emissions have an additional enhancement in their jovicentric longitude around $60^{\circ}$.

A possible reason of the CML extent and the enhancement of occurrence in the moon jovicentric longitude for the south components could be observational. Indeed Marques et al. [2017] did their catalog with the Nancay Decameter Array (NDA), observing only above $10 \mathrm{MHz}$, thus missing a part of the arcs. Furthermore, the range of jovicentric latitudes is different for the NDA and Voyager and Cassini, which could affect the visibility of the emissions [see Galopeau and Boudjada, 2016; Louis et al., 2017] (Section 2.1). A study of the lo-DAM with the Cassini/RPWS data for comparison will be interesting, but it is beyond the scope of this study.

Wannawichian et al. [2010] investigated the variation of the auroral UV footprint brightness of the Galilean moons as a function of the longitude. For lo they observed two peaks where the UV footprints are brighter: around $100^{\circ}$ and $280^{\circ}$ of lo jovicentric longitude. According to Marques et al. [2017] the radio emission activity has a peak around lo jovicentric longitudes $165^{\circ}$ and $220^{\circ}$. For Ganymede and Europa less data are available, but the brightest footprints were seen around $100^{\circ}$. In our study we found more Ganymede events around Ganymede jovicentric longitude $\sim 40^{\circ}$ (southern events) and $\sim 250^{\circ}$ (northern events) and more Europa events around Europa jovicentric longitude $\sim 65^{\circ}$ (southern events) and $\sim 250^{\circ}$ (northern events). At this point we do not have enough data to draw any conclusion about (anti-) correlation, or no correlation at all, between the DAM and UV emissions of Europa and Ganymede.

5.5. Discussion of Previous Tentative Detections of Jupiter-Ganymede and Jupiter-Europa Emissions Compared to the Galileo studies [Menietti et al., 1998a, 1998b; Higgins et al., 2006] we used a more extended data set (for Cassini almost 3000 Jupiter rotations [360 Europa rotations; 180 Ganymede rotations] against less than 1500 Jupiter rotations). The Cassini frequency range is also larger than that used for the above mentioned studies. Indeed, the previous studies were made at low frequency $(2.0-5.6 \mathrm{MHz})$. Only a small part of the whole emission lies in this frequency range (see Tables 1-3).

The study of Menietti et al. [1998a] focuses on two isolated arcs which could be consistent with Ganymedeinduced radio emissions. We computed ExPRES simulations corresponding to the Galileo trajectory. The EXPRES simulations did not show arcs related to Ganymede-induced radio emissions in a time window of $24 \mathrm{~h}$ around the arcs detected by Menietti et al. [1998a]. Furthermore, the Ganymede phase for these two arcs do not match the range of Ganymede phase described Table 5, thus these arcs cannot be associated with Ganymede.

Menietti et al. [1998b] and Hospodarsky et al. [2001] detected an enhancement in the occurrence probability for Ganymede-induced emissions near Ganymede phases $80^{\circ}$ and $245^{\circ}$ using a sinusoidal fit. Their studies showed a first hint of Ganymede's influence on the Jovian decametric emissions, with a first indication of the active Ganymede phases. Our results agree with theirs, with a more complete coverage of the Ganymede emissions in Ganymede phase and CML.

Higgins [2007] used the Voyager occurrence probability between 2.1 and $5.8 \mathrm{MHz}$ to propose Phase versus CML regions of maximum occurrence for the Galilean satellites. Compared to our study, Higgins [2007] used only the frequency range between 2.1 and $5.8 \mathrm{MHz}$, instead of the entire frequency range (extending up to $40 \mathrm{MHz}$ ). In our results (see Tables 1-3), except for the $\mathrm{C}$ component, the minimum value of the median minimum frequency of Europa and Ganymede emissions observed with the Voyager data is $7.00 \mathrm{MHz}$. Even if the peak of emission occurrence is minor, and fitted with a sinusoidal, for Europa, our results include theirs, and we can determine that the enhancement in emission occurrence they saw between $290^{\circ}$ and $350^{\circ}$ of CML and between $250^{\circ}$ and $280^{\circ}$ of Europa phase is included in the area of the Europa-A component, and the enhancement in emission occurrence they saw in the same $C M L$ range and between $85^{\circ}$ and $115^{\circ}$ of Europa phase is included in the area of the Europa-D component. 
On the other hand, our results do not include their results for Ganymede. They fitted an enhancement of occurrence emissions at Ganymede phases between $55^{\circ}$ and $85^{\circ}$, which would imply a large lead angle. The second peak of emission occurrence is detected at Ganymede phase between $305^{\circ}$ and $335^{\circ}$. Because of the complex morphology of the magnetic field, emissions could still be seen when the moon is geometrically a few degrees behind Jupiter. Here the region of phases given by Higgins [2007] means that the emissions are still visible from $30^{\circ}$ to $60^{\circ}$ after the geometrical disappearance of the moon, which cannot be explained by the lead angle at these phases. In summary, our results include those of Higgins [2007] for Europa and do not agree with those of Higgins [2007] for Ganymede.

\section{Conclusions and Perspectives}

Using EXPRES we made a catalog of simulations of all the Galilean moons induced radio emissions. We then studied almost 4 years of Cassini observations, and 6 months of Voyager observations, during their flyby of Jupiter, compared them with the simulations, and identified clear detections of Europa (29 Europa-A, 16 Europa-B, 31 Europa-C, and 54 Europa-D) and Ganymede (21 Ganymede-A, 18 Ganymede-B, 22 Ganymede-C, and 35 Ganymede-D) induced radio emissions. The number of false detections is likely to be low.

The shape of Europa-DAM and Ganymede-DAM emissions is nearly the same as that of the lo-DAM emissions. The median spectral and temporal properties of the Europa-DAM and Ganymede-DAM emissions are summarized in Table 3, the maximum occurrence regions of Europa and Ganymede DAM emissions are described in Tables 4 and 5, respectively and plotted in Figure 6. The active jovicentric longitudes of Europa and Ganymede are plotted in Figure 7 and described in detail in Tables 4 and 5.

Stereoscopic observations with ground-based radio telescopes will help to assess the short-term variability of these emissions, and a long-term catalog (e.g., with Wind and Stereo) will help us to assess long-term variability.

\section{Appendix A: Satellite Phase Versus Observer CML Diagrams of the ExPRES Simulations for Europa and Ganymede}

Figure A1 displays phase versus CML diagrams of the Cassini (Figures A1a-A1h) (year 2000) and Voyager simulations (Figures A1i-A1p) (including DoY 001 to 090, 1979 for Voyager 1 and DoY 120 to 210, 1979 for Voyager 2, corresponding to the intervals where we detect events related to Europa and Ganymede) for Europa (Figures A1a, A1e, A1i, and A1m) and Ganymede-induced emissions (Figures A1c, A1g, A1k, and A1o). The left panels represent the simulations corresponding to the $V E$ and $V L$ arcs (Figures $A 1 a, A 1 c, A 1 i$, and $A 1 k$ ), and the right panels represent the whole simulations (Figures $A 1 \mathrm{e}, \mathrm{A} 1 \mathrm{~g}, \mathrm{~A} 1 \mathrm{~m}$, and $\mathrm{A} 1 \mathrm{o}$ ). The corresponding occurrence sorted by the moons' phase are plotted on the right of the Phase versus CML diagrams. The northern emissions are represented in blue and the southern ones in red.

With the selection criteria on the VE and VL arcs (left panels) we see that the simulations are grouped in phase - as the diagram of the detections - but not in CML, unlike in Figure 6. Thus, the distribution must have a physical origin other than geometrical, which is corroborated by the discussion in section 2.1.

The right panels show that for Cassini (Figures $\mathrm{A} 1 \mathrm{a}-\mathrm{A} 1 \mathrm{~h}$ ), the distribution in phase does not depend on the selection criteria whether the arc is VE or VL, because the modulation of the emission seen in Figure 1 occurs at frequencies higher than $16 \mathrm{MHz}$. We see a minor contribution of the oscillations of the north emissions, because some of them happens at frequency under $16 \mathrm{MHz}$ (see Figure 1).

For Voyager (Figures $\mathrm{A} 1 \mathrm{i}-\mathrm{A} 1 \mathrm{p})$ we see that without the selection criteria whether the arc is VE or VL (Figures $A 1 n$ and $A 1 p$ ) there are emissions between $120^{\circ}$ and $260^{\circ}$. But it remains that the emissions are mostly grouped at phases $120^{\circ}$ and $260^{\circ}$ (Figures $A 1 n$ and $A 1 p$ compared to Figures $A 1 j$ and $A 1 \mathrm{l}$ ). Thus, we can conclude that the distribution in phase mostly does not depend on the selection criteria whether the arc is VE or VL.

The modulation in phase in the diagram is likely due to the modulation of the magnetic field, which deforms the magnetic field lines. 


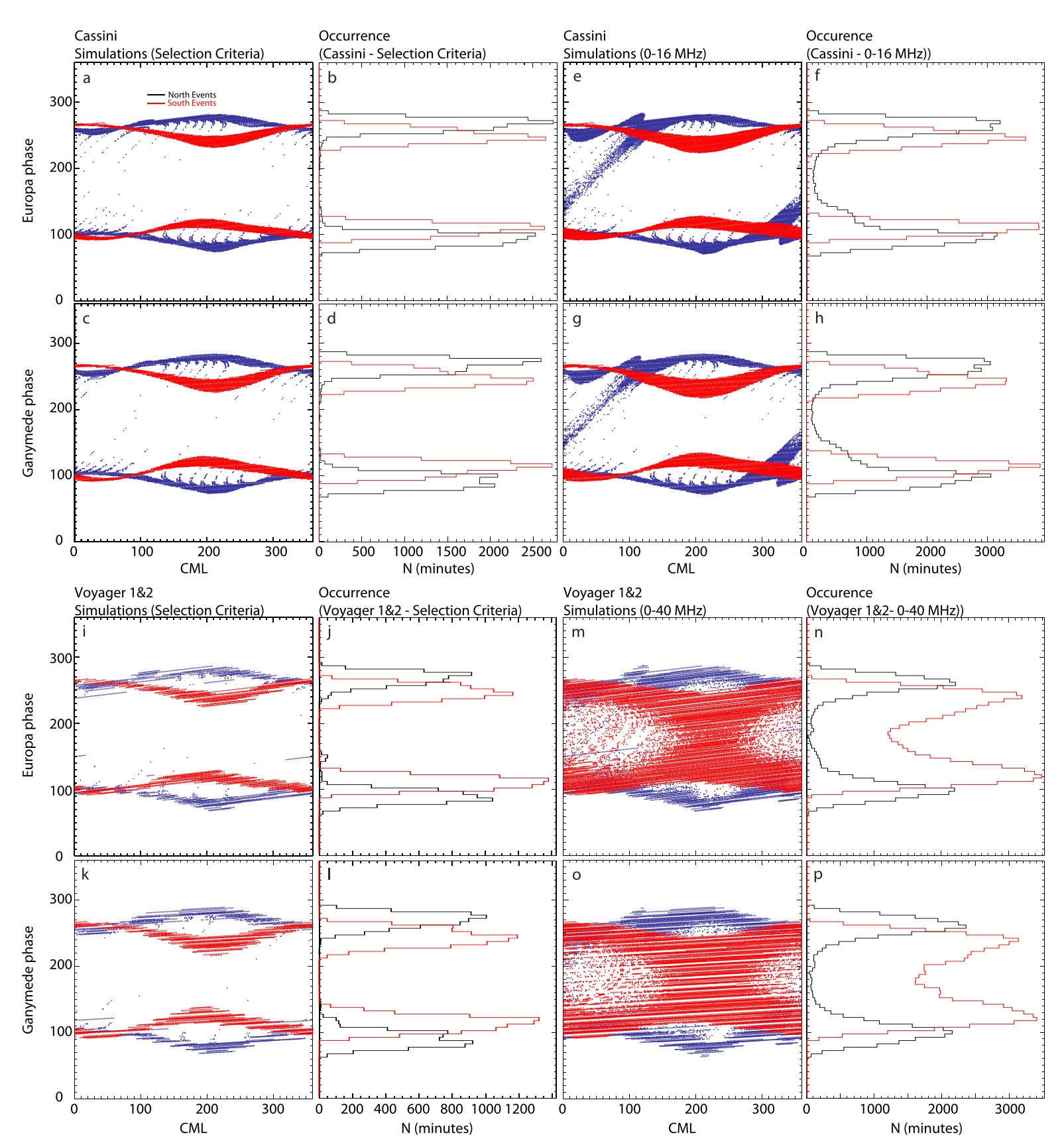

Figure A1. Satellite phase versus observer CML diagrams of the ExPRES simulations for $(a, e, i, m)$ Europa and $(c, g, k, 0)$ Ganymede and the corresponding occurrence in (b,f,j,n and d,h,i,p, respectively) phase for the Cassini (Figures A1a-A1h) (year 2000) and Voyager 1 and 2 spacecraft (Figures A1i-A1p) (from day 1 to day 90 of 1979 for Voyager 1 and from day 120 to day 210 of 1979 for Voyager 2). Figures A1a-A1d and A1i-A1I represent the VE and VL arcs, and Figures $\mathrm{A} 1 \mathrm{e}-\mathrm{A} 1 \mathrm{~h}$ and $\mathrm{A} 1 \mathrm{~m}-\mathrm{A} 1 \mathrm{p}$ the entire arcs. The north events are represented in blue and the southern ones in red.

\section{Appendix B: Effects of the Emission Cone Opening Angle $\theta$}

As explained in section 5.1, the position of the emissions in time and frequency depends on the value of $\theta$. Figure B1 compares the intensity flux of a Cassini observation (Figure B1a) to three simulations (Figure B1b) of the Europa-C emission, presented in Figure 2, with different values of $\theta$ :

1. The one in blue dotted line is the one used in the study to detect an Europa-C arc in the Cassini data (see Figure 2) with a $\theta$ calculated from a loss cone electron distribution function;

2. The one in light blue dotted line is a simulation with a constant $\theta=60^{\circ}$, which could correspond to an emission with strong refraction effects; 


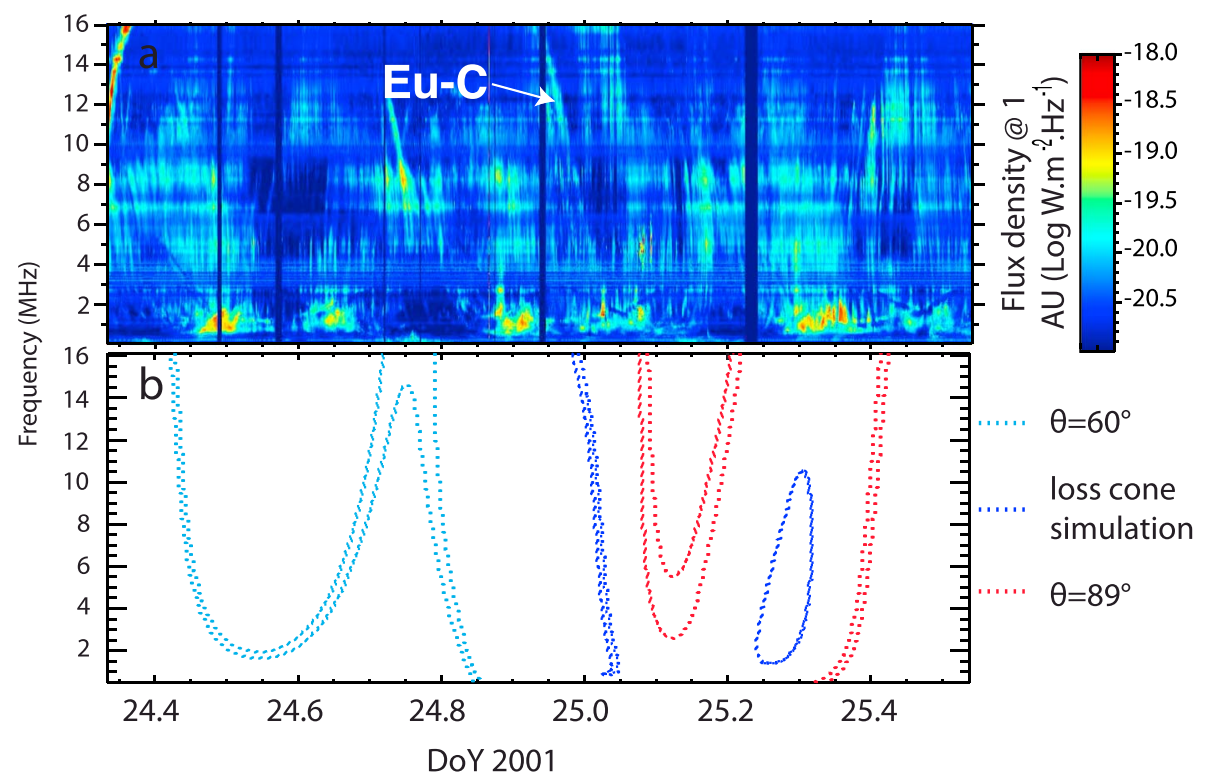

Figure B1. Cassini observation of a Europa arc (VL and LH polarized) and different simulations of this arc. (a) Intensity flux and (b) three simulations with different values of $\theta$. The one in blue dotted line uses a $\theta$ calculated from a loss cone electron distribution function, the one in light blue dotted line uses a constant $\theta=60^{\circ}$, and the one in red dotted line a constant $\theta=89^{\circ}$.

3. The one in red dotted line is a simulation with a constant $\theta=89^{\circ}$, which is close to a perpendicular emission.

We see the impact of the $\theta$ value, with a difference in time between 1 and $5 \mathrm{~h}$ around the simulation calculated from a loss cone electron distribution function, and a large difference in the shape of the arc.

For one value of the electron energy and one magnetic field line the simulation with the loss cone distribution function gives values of $\theta$ fixed by frequency [see Hess et al., 2008, Figure 2b]: the emission cone angle $\theta$ is oblique, and decreases with increasing $f$. The variation of $\theta$ may have three different causes: (1) the presence of a lead angle that make the emission appear earlier for a VE arc (as if $\theta$ was greater) or disappear earlier for a VL arc (as if $\theta$ was lower); (2) refraction effects that oblate the emission cone [see Galopeau and Boudjada, 2016]; (3) a wrong estimate of $\theta$ modifies the occurrence in time, depending on the rotation speed of the moon $\left(\omega_{\text {moon }}\right.$ ). A wrong estimate of $\theta$ of $10^{\circ}$ corresponds to a shift of: $\simeq 71 \mathrm{~min}$ for lo; $\simeq 140 \mathrm{~min}$ for Europa; and $\simeq 280 \mathrm{~min}$ for Ganymede. The selection criteria in time have been chosen taking into account the above three possibilities.

\section{Acknowledgments}

We thank the anonymous referees for their thorough review. C.L. thanks E. Bonnassieux for proofreading this paper. This research has made use of IMCCE's Miriade VO tool. The ExPRES tool and the Kronos database are part of the MASER (Measurement, Analysis, and Simulations of Emissions in Radio), which is supported by PADC (Paris Astronomical Data Centre). Coauthors were supported by CNES (Centre National d'Etudes Spatiale). This work has been done within the LABEX Plas@par project and received financial state aid managed by the "Agence Nationale de la Recherche," as part of the program "Investissements d'avenir" under the reference ANR-11-IDEX-0004-02.

\section{References}

Bagenal, F. (1994), Empirical model of the lo plasma torus: Voyager measurements, J. Geophys. Res., 99(A6), 11,043-11,062. Bagenal, F., et al. (2014), Magnetospheric science objectives of the Juno mission, Space Sci. Rev., 1-69, doi:10.1007/s11214-014-0036-8. Bigg, E. K. (1964), Influence of the satellite lo on Jupiter's decametric emission, Nature, 203, 1008-1010, doi:10.1038/2031008a0.

Bonfond, B., S. Hess, F. Bagenal, J.-C. Grard, D. Grodent, A. Radioti, J. Gustin, and J. T. Clarke (2013), The multiple spots of the Ganymede auroral footprint, Geophys. Res. Lett., 40, 4977-4981, doi:10.1002/grl.50989.

Carr, T. D., M. D. Desch, and J. K. Alexander (1983), Phenomenology of magnetospheric radio emissions, in Physics of the Jovian Magnetosphere, vol. 3, edited by A. J. Dessler, pp. 226-284, Cambridge Planet. Sci. Ser., Cambridge Univ. Press, New York.

Cecconi, B., et al. (2012), Natural radio emission of Jupiter as interferences for radar investigations of the icy satellites of Jupiter, Planet. Space Sci., 61, 32-45.

Cecconi, B., A. Preuvot, L. Lamy, P. Zarka, C. Louis, S. L. G. Hess, D. R. Evans, and D. Boucon (2017), Refurbishing Voyager 1 \& 2 Planetary Radio Astronomy (PRA) data, in Planetary Radio Emissions VIII, edited by G. Fischer, Austrian Acad. Press, Vienna.

Clarke, J. T., et al. (1998), Hubble space telescope imaging of Jupiter's magnetosphere, Science, 262, 1035-1038.

Clarke, J. T., et al. (2002), Ultraviolet emissions from the magnetic footprints of lo, Ganymede and Europa on Jupiter, Nature, 415, 997-1000. Connerney, J. E. P., M. H. Acuna, and N. F. Ness (1981), Modeling the Jovian current sheet and inner magnetosphere, J. Geophys. Res., 86, 8370-8384, doi:10.1029/JA086iA10p08370.

Connerney, J. E. P., R. Baron, T. Satoh, and T. Owen (1993), Images of excited $H_{3}$ at the foot of the lo flux tube in Jupiter's atmosphere, Science, 262(5136), 1035-1038.

Galopeau, P. H. M., and M. Y. Boudjada (2016), An oblate beaming cone for lo-controlled Jovian decameter emission, J. Geophys. Res. Space Physics, 121, 3120-3138, doi:10.1002/2015JA021038.

Gurnett, D. A., et al. (2004), The Cassini radio and plasma wave investigation, Space Sci. Rev., 114, 395-463.

Hess, S., B. Cecconi, and P. Zarka (2008), Modeling of lo-Jupiter decameter arcs, emission beaming and energy source, Geophys. Res. Lett., 35, L13107, doi:10.1029/2008GL033656. 
Hess, S. L. G., and P. Zarka (2011), Modeling the radio signature of the orbital parameters, rotation, and magnetic field of exoplanets, Astro. Astrophys., 531, A29.

Hess, S. L. G., A. Pétin, P. Zarka, B. Bonfond, and B. Cecconi (2010), Lead angles and emitting electron energies of lo-controlled decameter radio arcs, Planet. Space Sci., 58, 1188-1198.

Hess, S. L. G., B. Bonfond, P. Zarka, and D. Grodent (2011a), Model of the Jovian magnetic field topology constrained by the lo auroral emissions, J. Geophys. Res., 116, A05217, doi:10.1029/2010JA016262.

Hess, S. L. G., P. A. Delamere, V. Dols, and L. C. Ray (2011b), Comparative study of the power transferred from satellite-magnetosphere interactions to auroral emissions, J. Geophys. Res., 116, A01202, doi:10.1029/2010JA015807.

Hess, S. L. G., E. Echer, and P. Zarka (2012), Solar wind pressure effects on Jupiter decametric radio emissions independent of lo, Planet. Space Sci., 70, 114-125.

Hess, S. L. G., E. Echer, P. Zarka, L. Lamy, and P. A. Delamere (2014), Multi-instrument study of the Jovian radio emissions triggered by solar wind shocks and inferred magnetospheric subcorotation rates, Planet. Space Sci., 99, 136-148.

Hess, S. L. G., B. Bonfond, Bagenal F., and L. Lamy (2017), A model of the Jovian internal field derived from in-situ and auroral constraints, in Planetary Radio Emissions VIII, edited by G. Fischer et al., Austrian Acad. Press, Vienna, Austria.

Higgins, C. A. (2007), Satellite control of Jovian 2-6 MHz radio emission using Voyager data, J. Geophys. Res., 112, A05213, doi:10.1029/2006JA012100.

Higgins, C. A., J. D. Menietti, and I. W. Christopher (2006), Europa control of Jovian radio emission: A Galileo study, Geophys. Res. Lett., 33, L14110, doi:10.1029/2006GL026218.

Hilgers, A. (1992), The auroral radiating plasma cavities, Geophys. Res. Lett., 19(3), 237-240.

Hinson, D. P., J. D. Twicken, and E. T. Karayel (1998), Jupiter's ionosphere: New results from Voyager 2 radio occultation measurements, J. Geophys. Res., 103(A5), 9505-9520.

Hospodarsky, G. B., I. W. Christopher, J. D. Meniett, W. S. Kurth, D. A. Gurnett, T. F. Averkamp, J. B. Groene, and P. Zarka (2001), Control of Jovian radio emissions by the Galilean moons as observed by Cassini and Galileo, in Proceedings of the 5th International Workshop held at Graz Austria, edited by H. O. Rucker, M. L. Kaiser, and Y. Leblanc, pp. 155-164, Planetary Radio Emissions V. Austrian Acad. Sci. Press, Vienna.

Kurth, W. S., D. A. Gurnett, and J. D. Menietti (2000), The influence of the Galilean satellites on radio emissions from the Jovian system, in Radio Astronomy at Long Wavelengths, Geophys. Monogr., vol. 119, edited by R. G. Stone et al., pp. 213-225, AGU, Washington, D. C.

Lamy, L., P. Zarka, B. Cecconi, R. Prangé, W. S. Kurth, and D. A. Gurnett (2008a), Saturn kilometric radiation: Average and statistical properties, J. Geophys. Res., 113, A07201, doi:10.1029/2007JA012900.

Lamy, L., P. Zarka, B. Cecconi, S. Hess, and R. Prangé (2008b), Modeling of Saturn kilometric radiation arcs and equatorial shadow zone, J. Geophys. Res., 113, A10213, doi:10.1029/2008JA013464.

Lamy, L., R. Prangé, W. Pryor, J. Gustin, S. V. Badman, H. Melin, T. Stallard, D. G. Mitchell, and P. C. Brandt (2013), Multispectral simultaneous diagnosis of Saturn's aurorae throughout a planetary rotation, J. Geophys. Res. Space Physics, 118, 4817-4843, doi:10.1002/jgra.50404.

Louis, C. K., et al. (2017), lo-Jupiter decametric arcs observed by Juno/Waves compared to ExPRES simulations, Geophys. Res. Lett., 44, doi:10.1002/2017GL073036.

Marques, M., P. Zarka, E. Echer, V. B. Ryabov, M. V. Alves, L. Denis, and A. Coffre (2017), Statistical analysis of 26 years of observations of decametric radio emissions from Jupiter, Astron. Astrophys., 604, A17.

Menietti, J. D., D. A. Gurnett, W. S. Kurth, J. B. Groene, and L. J. Granroth (1998a), Galileo direction finding of Jovian radio emissions, J. Geophys. Res., 103(E9), 20001-20010, doi:10.1029/97JE03555.

Menietti, J. D., D. A. Gurnett, W. S. Kurth, and J. B. Groene (1998b), Control of Jovian radio emission by Ganymede, Geophys. Res. Lett., 25(23), $4281-4284$.

Menietti, J. D., D. A. Gurnett, and I. Christopher (2001), Control of Jovian radio emission by Callisto, Geophys. Res. Lett., 28, 3047-3050.

Mottez, F., S. Hess, and P. Zarka (2010), Explanation of dominant oblique radio emission at Jupiter and comparison to the terrestrial case, Planet. Space Sci., 58, 1414-1422.

Neubauer, F. M. (1980), Nonlinear standing Alfvén wave current system at lo: Theory, J. Geophys. Res., 85(13), $1171-1178$.

Prangé, R., D. Rego, D. Southwood, P. Zarka, S. Miller, and W. Ip (1996), Rapid energy dissipation and variability of the lo-Jupiter electrodynamic circuit, Nature, 379, 323-325.

Ray, L. C., and S. Hess (2008), Modelling the lo-related DAM emission by modifying the beaming angle, J. Geophys. Res., 113, A11218, doi:10.1029/2008JA013669.

Saur, J., F. M. Neubaeur, J. E. P. Connerney, P. Zarka, and M. G. Kivelson (2004), Plasma interaction of lo with its plasma torus, in Jupiter: The Planet, Satellites and Magnetosphere, edited by F. Bagenal, T. E. Dowling, and W. B. Mckinnon, pp. 537-560, Cambridge Univ. Press, Cambridge, U. K.

Treumann, R. A. (2006), The electron-cyclotron maser for astrophysical application, Astron. Astrophys. Rev., 13, 229-315, doi:10.1007/s00159-006-0001-y.

Wannawichian, S., J. T. Clarke, and J. D. Nichols (2010), Ten years of Hubble space telescope observations of the variation of the Jovian satellites auroral footprint brightness, J. Geophys. Res., 115, A02206, doi:10.1029/2009JA014456.

Zarka, P. (1998), Auroral radio emissions at the outer planets: Observations and theories, J. Geophys. Res., 103(E9), 20,159-20,194.

Zarka, P. (2007), Plasma interactions of exoplanets with their parent star and associated radio emissions, Planet. Space Sci., 55, 598-617.

Zarka, P., J. Queinnec, and F. J. Crary (2001), Low-frequency limit of Jovian radio emissions and implications on source locations and lo plasma wake, Planet. Space Sci., 49, 1137-1149. 